\title{
MACROINVERTEBRADOS DEL NORTE DE SANTANDER
}

Por:

M.Sc. Mariorie Josefina Sánchez Herrera'

Mylenda J. Avendaño Sánchez²

\section{RESUMEN}

El presente artículo tiene como fin reportar las morfofamilias de macroinvertebrados capturadas e identificadas en diferentes puntos de las principales cuencas del departamento Norte de Santander: Pamplonita, Zulia, Algodonal y la Subcuenca del Táchira; contribuyendo al conocimiento de la biodiversidad acuática de la región.

Palabras claves: BMWP, Bioindicadores, Biodiversidad, Macroinvertebrados

\section{ABSTRACT}

The aim of this paper is to report the morfofamilies of macroinvertebrates captured and identified in different points of the main basins of the rivers Pamplonita, Zulia, Algodonal and Táchira in the department of Norte de Santander, contributing to the knowledge of the aquatic biodiversity.

Key words: BMWP, Bioindicators, Biodiversity, Macroinvertebrates.

\section{INTRODUCCIÓN}

Los Macroinvertebrados se han convertido paulatinamente en una herramienta práctica para la determinación de la calidad biológica, esto se ha podido lograr gracias a la capacidad que tienen estos organismos de mantener una estrecha relación con las condiciones del entorno, es decir son sensibles a pequeñas alteraciones de los parámetros fisicoquímicos del agua, esto representado en la disminución o aumento de la diversidad en un punto de muestreo. Además la aplicación de este tipo de técnicas permite una reducción considerable en los costos de inversión necesarios para determinar la calidad del agua. Los muestreos se han venido realizando desde 1999 en el marco del desarrollo de varios proyectos tales como: Macroinvertebrados acuáticos como bioindicadores de la calidad del agua de la zona media del río Pamplonita, Estudio Limnológico de la Zona Alta del río Pamplonita, Diseño del Plan de Monitoreo de la calidad y la cantidad del recurso hídrico de las cuencas de los río Zulia, Pamplonita, Algodonal y la subcuenca del río Táchira del departamento Norte de Santander patrocinados por el Fondo de Investigaciones Universitarias FINU - UFPS, Facultad en Ciencias Agrarias y del Ambiente, Facultad de Ciencias Básicas de la Universidad Francisco de Paula Santander y CORPONOR.

\section{MATERIALES Y MÉTODOS}

Cuadro 1. Matriz de procedimientos.

\begin{tabular}{|c|c|c|}
\hline ANÁLISIS BIOLÓGICO & MÉTODO & DESCRIPCIÓN \\
\hline $\begin{array}{l}\text { Captura de los organismos } \\
\text { para los a nálisis } \\
\text { cualitativos y cuantitativos } \\
\text { de los Macroinvertebrados } \\
\text { presentes en el agua } \\
\text { característicos de la } \\
\text { Bioindicación }\end{array}$ & $\begin{array}{l}\text { Muestra } \\
\text { Compuesta } \\
\text { Mallas: } \\
>\text { Pantalla } \\
\text { D D'Net }\end{array}$ & $\begin{array}{l}\text { El muestreo cualitativo se realiza con las redes de } \\
\text { pantalla y D’Net. La muestra es combinada } \\
\text { incluyendo sedimentos pedregosos, arenosos, } \\
\text { vegetación circundante, rápidosy remansos. } \\
\text { Una vez obtenidas las muestras, se colocan en bolsas } \\
\text { plásticas adicionándole solución FAGA 1:1 (Formol, } \\
\text { alcohol, glicerina y agua) para su preservación; se } \\
\text { rotula la muestra } \\
\text { En el laboratorio cada muestra es vaciada y separada } \\
\text { en cajas petri, se procede a limpiar los restos de } \\
\text { material orgánico inorgánico } \\
\text { La identificación de las morfofamilias se realiza } \\
\text { teniendo como patrones de referencia las claves } \\
\text { taxonómicas; la descripción se realiza con } \\
\text { estereoscopio Wild M3, oculares } 25 x y 10 x\end{array}$ \\
\hline
\end{tabular}

Para la identificación de los organismo, se utilizaron las claves taxonómicas para Macroinvertebrados, tales como:

\footnotetext{
' Licenciada en Biología y Química U. de Pamplona, M.Sc. en Biología U. de los Andes. Docente Emerito Universidad Francisco de Paula Santander, Directora Grupo de Investigación GUIA - UFPS. marjoriejosefina@latinmail.com

2 Estudiante X Semestre Ingeniería Agroindustrial - UFPS
} 
- Edmonson

- Roldan

- Mc. Cafferty

- Brinkhurst y Marchese

- NeedHam y NeedHamn

- Curso de Insectos Inmaduros UNIVALLE

- H.R Fernández y E. Domínguez

\section{RESULTADOS}

A continuación se relacionan las familias identificadas y en que puntos fueron capturadas en las cuatro principales cuencas del Departamento Norte de Santander, las fotografías muestran en su mayoría los estadíos inmaduros de los individuos con algunas excepciones como es el caso del orden Tubificida.

Orden: Acarida

\begin{tabular}{|c|c|c|c|c|c|c|c|c|c|}
\hline \multirow{2}{*}{\multicolumn{2}{|c|}{ FAMILIA }} & \multicolumn{2}{|c|}{ Cuenca río Zulia } & \multicolumn{2}{|c|}{$\begin{array}{l}\text { Cuenca río } \\
\text { Pamplonita }\end{array}$} & \multicolumn{2}{|c|}{$\begin{array}{l}\text { Cuenca río } \\
\text { Algodonal }\end{array}$} & \multicolumn{2}{|c|}{$\begin{array}{c}\text { Cuenca río } \\
\text { Táchira }\end{array}$} \\
\hline & & msnm & ${ }^{\circ} \mathrm{C}$ & msnm & ${ }^{\circ} \mathrm{C}$ & msnm & ${ }^{\circ} \mathrm{C}$ & msnm & ${ }^{\circ} \mathrm{C}$ \\
\hline 1 & $\begin{array}{l}\text { Hydrachnidia } \\
\text { (Hidracaridos) }\end{array}$ & $\begin{array}{c}1200 \\
925\end{array}$ & $\begin{array}{l}21.8 \\
23.2\end{array}$ & $\begin{array}{c}2900 \\
2400 \\
719 \\
400 \\
250 \\
200 \\
160 \\
50\end{array}$ & $\begin{array}{c}11.5 \\
14.5 \\
24 \\
25 \\
33 \\
34.5 \\
30 \\
33.5\end{array}$ & 1233 & 22 & 900 & 35.5 \\
\hline
\end{tabular}

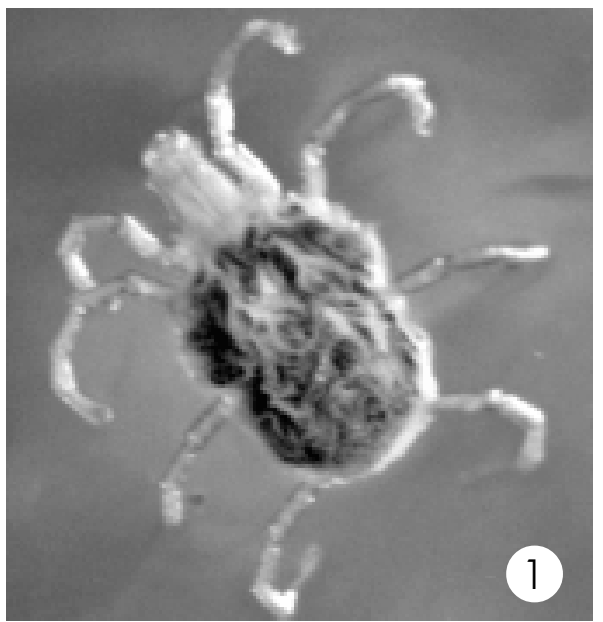

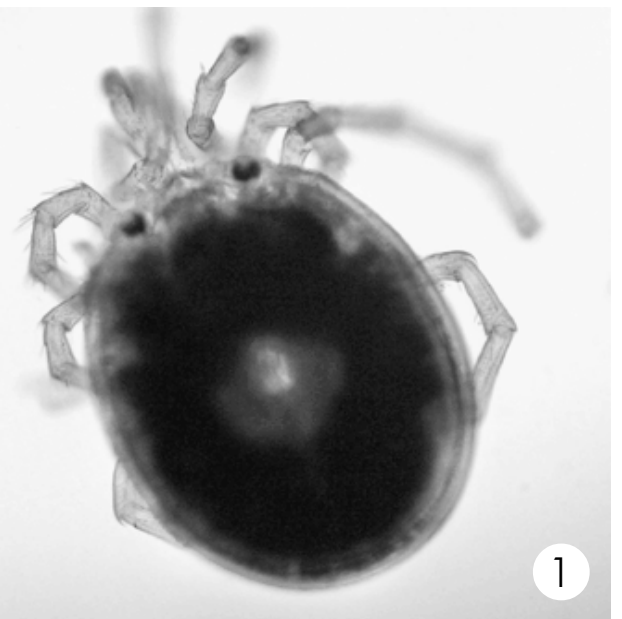

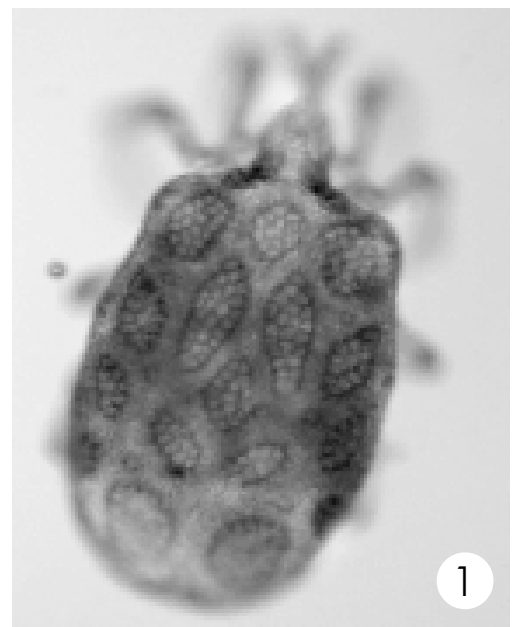

Revista Respuestas - Universidad Francisco de Paula Santander Año 10 No. 1 
Orden: Amphipoda

\begin{tabular}{|l|l|c|c|c|c|c|c|c|c|}
\hline \multirow{2}{*}{ FAMILIA } & \multicolumn{2}{|c|}{ Cuenca río Zulia } & \multicolumn{2}{c|}{$\begin{array}{c}\text { Cuenca río } \\
\text { Pamplonita }\end{array}$} & \multicolumn{2}{c|}{$\begin{array}{c}\text { Cuenca río } \\
\text { Algodonal }\end{array}$} & \multicolumn{2}{c|}{$\begin{array}{c}\text { Cuenca río } \\
\text { Táchira }\end{array}$} \\
\cline { 2 - 9 } & msnm & ${ }^{\circ} \mathrm{C}$ & $\mathrm{msnm}$ & ${ }^{\circ} \mathrm{C}$ & $\mathrm{msnm}$ & ${ }^{\circ} \mathrm{C}$ & $\mathrm{msnm}$ & ${ }^{\circ} \mathrm{C}$ \\
\hline \multirow{2}{*}{2} & Hyalellidae & & & 2900 & 11.5 & & & & \\
& & & 2800 & 12.5 & & & & \\
& & & 2120 & 17.6 & & & & \\
& & & 1045 & 19.5 & & & & \\
\hline
\end{tabular}
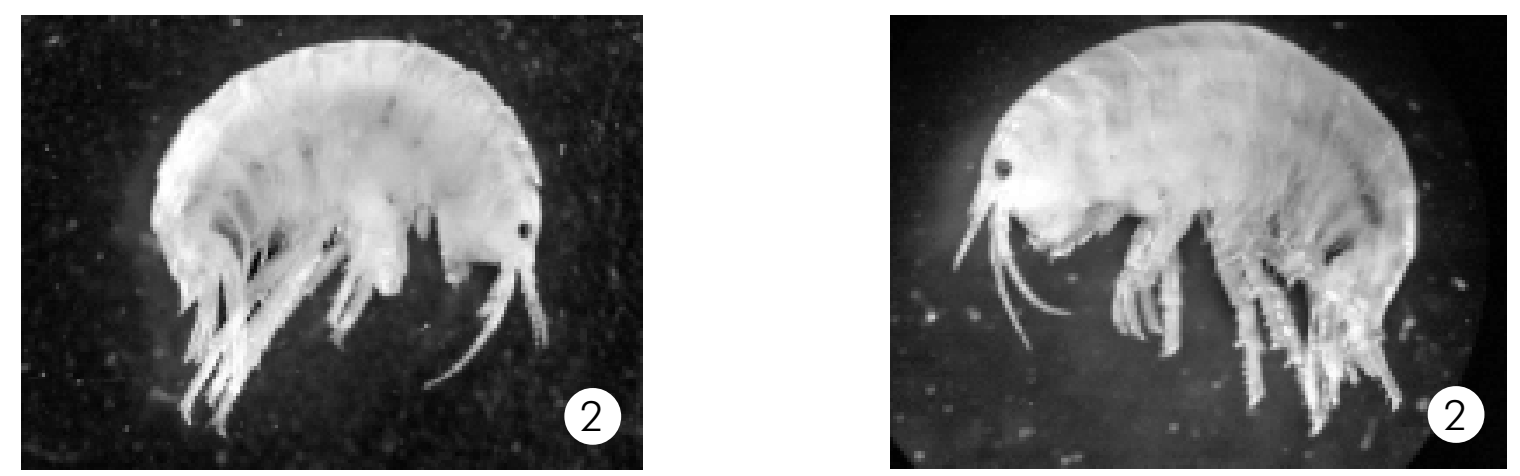

Orden: Basommatophora

\begin{tabular}{|c|c|c|c|c|c|c|c|c|c|}
\hline \multirow{2}{*}{\multicolumn{2}{|c|}{ FAMILIA }} & \multicolumn{2}{|c|}{ Cuenca río Zulia } & \multicolumn{2}{|c|}{$\begin{array}{l}\text { Cuenca río } \\
\text { Pamplonita }\end{array}$} & \multicolumn{2}{|c|}{$\begin{array}{l}\text { Cuenca río } \\
\text { Algodonal }\end{array}$} & \multicolumn{2}{|c|}{$\begin{array}{c}\text { Cuenca río } \\
\text { Táchira }\end{array}$} \\
\hline & & msnm & ${ }^{\circ} \mathrm{C}$ & msnm & ${ }^{\circ} \mathrm{C}$ & msnm & ${ }^{\circ} \mathrm{C}$ & $\mathrm{msnm}$ & ${ }^{\circ} \mathrm{C}$ \\
\hline 3 & Physidae & $\begin{array}{l}1298 \\
272\end{array}$ & $\begin{array}{l}18.7 \\
23.7\end{array}$ & $\begin{array}{l}2400 \\
2249 \\
2120 \\
1045 \\
719 \\
400 \\
250 \\
160 \\
100\end{array}$ & $\begin{array}{l}14.5 \\
14.5 \\
17.5 \\
19.5 \\
24 \\
25 \\
33 \\
30 \\
32\end{array}$ & & & 1200 & 26.5 \\
\hline 3 & Lymnaeidae & & & 2400 & 14.5 & & & & \\
\hline 4 & Planorbidae & & & 2400 & 14.5 & & & & \\
\hline 5 & Ancylidae & & & $\begin{array}{l}400 \\
250 \\
160 \\
100\end{array}$ & $\begin{array}{l}25 \\
33 \\
30 \\
32 \\
\end{array}$ & & & & \\
\hline
\end{tabular}
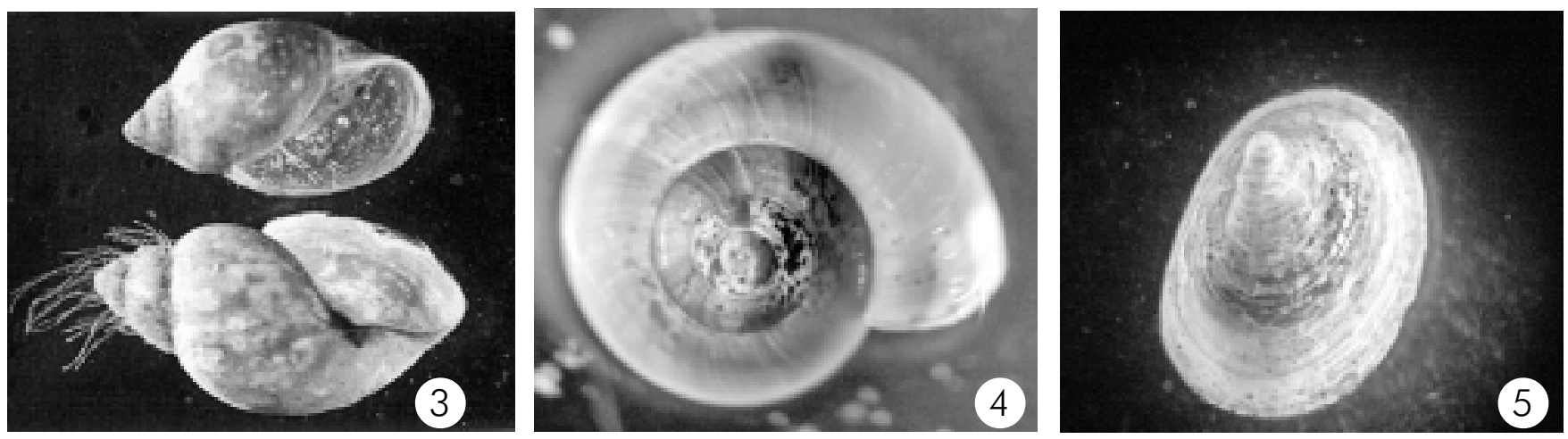
Macroinvertebrados del Norte de Santander

\section{Orden: Coleoptera}

\begin{tabular}{|c|c|c|c|c|c|c|c|c|c|}
\hline \multirow{2}{*}{\multicolumn{2}{|c|}{ FAMILIA }} & \multicolumn{2}{|c|}{ Cuenca río Zulia } & \multicolumn{2}{|c|}{$\begin{array}{l}\text { Cuenca río } \\
\text { Pamplonita }\end{array}$} & \multicolumn{2}{|c|}{$\begin{array}{l}\text { Cuenca río } \\
\text { Algodonal }\end{array}$} & \multicolumn{2}{|c|}{$\begin{array}{l}\text { Cuenca río } \\
\text { Táchira }\end{array}$} \\
\hline & & msnm & ${ }^{\circ} \mathrm{C}$ & $\mathrm{msnm}$ & ${ }^{\circ} \mathrm{C}$ & $\mathrm{msnm}$ & ${ }^{\circ} \mathrm{C}$ & $\mathrm{msnm}$ & ${ }^{\circ} \mathrm{C}$ \\
\hline 6 & Hydrophilidae & & & $\begin{array}{l}400 \\
200 \\
160\end{array}$ & $\begin{array}{c}25 \\
34.5 \\
30\end{array}$ & & & & \\
\hline 7 & Ptilodactylidae & & & $\begin{array}{l}2900 \\
2800\end{array}$ & $\begin{array}{l}11.5 \\
12.5\end{array}$ & 1233 & 22 & & \\
\hline 8 & Elmidae & $\begin{array}{l}1298 \\
1200 \\
925 \\
904 \\
279 \\
272\end{array}$ & $\begin{array}{l}18.7 \\
21.8 \\
23.2 \\
24 \\
24 \\
23.7\end{array}$ & $\begin{array}{l}2900 \\
2800 \\
2314 \\
2120 \\
1045 \\
719 \\
400 \\
250 \\
200 \\
160 \\
100 \\
50\end{array}$ & $\begin{array}{l}11.5 \\
12.5 \\
14.5 \\
17.5 \\
19.5 \\
24 \\
25 \\
33 \\
34.5 \\
30 \\
32 \\
33.5\end{array}$ & $\begin{array}{l}1392 \\
1310 \\
1233 \\
1209 \\
970\end{array}$ & $\begin{array}{c}21 \\
20.2 \\
22 \\
24.8 \\
23.5\end{array}$ & $\begin{array}{l}1200 \\
900 \\
300 \\
250\end{array}$ & $\begin{array}{l}26.5 \\
35.5 \\
30.2 \\
33\end{array}$ \\
\hline 9 & Helodidae & & & $\begin{array}{l}2900 \\
2800 \\
2314\end{array}$ & $\begin{array}{l}11.5 \\
12.5 \\
14.5\end{array}$ & & & & \\
\hline 10 & Psephenidae & $\begin{array}{l}1200 \\
50\end{array}$ & $\begin{array}{l}21.8 \\
32\end{array}$ & $\begin{array}{l}1045 \\
719 \\
400 \\
250 \\
160\end{array}$ & $\begin{array}{l}19.5 \\
24 \\
25 \\
33 \\
30\end{array}$ & $\begin{array}{l}1379 \\
1233\end{array}$ & $\begin{array}{l}22 \\
22\end{array}$ & 300 & 30.2 \\
\hline 11 & Dytiscidae & & & 2120 & 17.5 & & & & \\
\hline 12 & Staphylinidae & & & 200 & 34.5 & & & & \\
\hline 13 & Leptinidae & & & 400 & 25 & & & & \\
\hline 14 & Coleoptera sp. & 279 & 24 & $\begin{array}{l}719 \\
400 \\
250 \\
160\end{array}$ & $\begin{array}{l}24 \\
25 \\
33 \\
30\end{array}$ & & & & \\
\hline
\end{tabular}
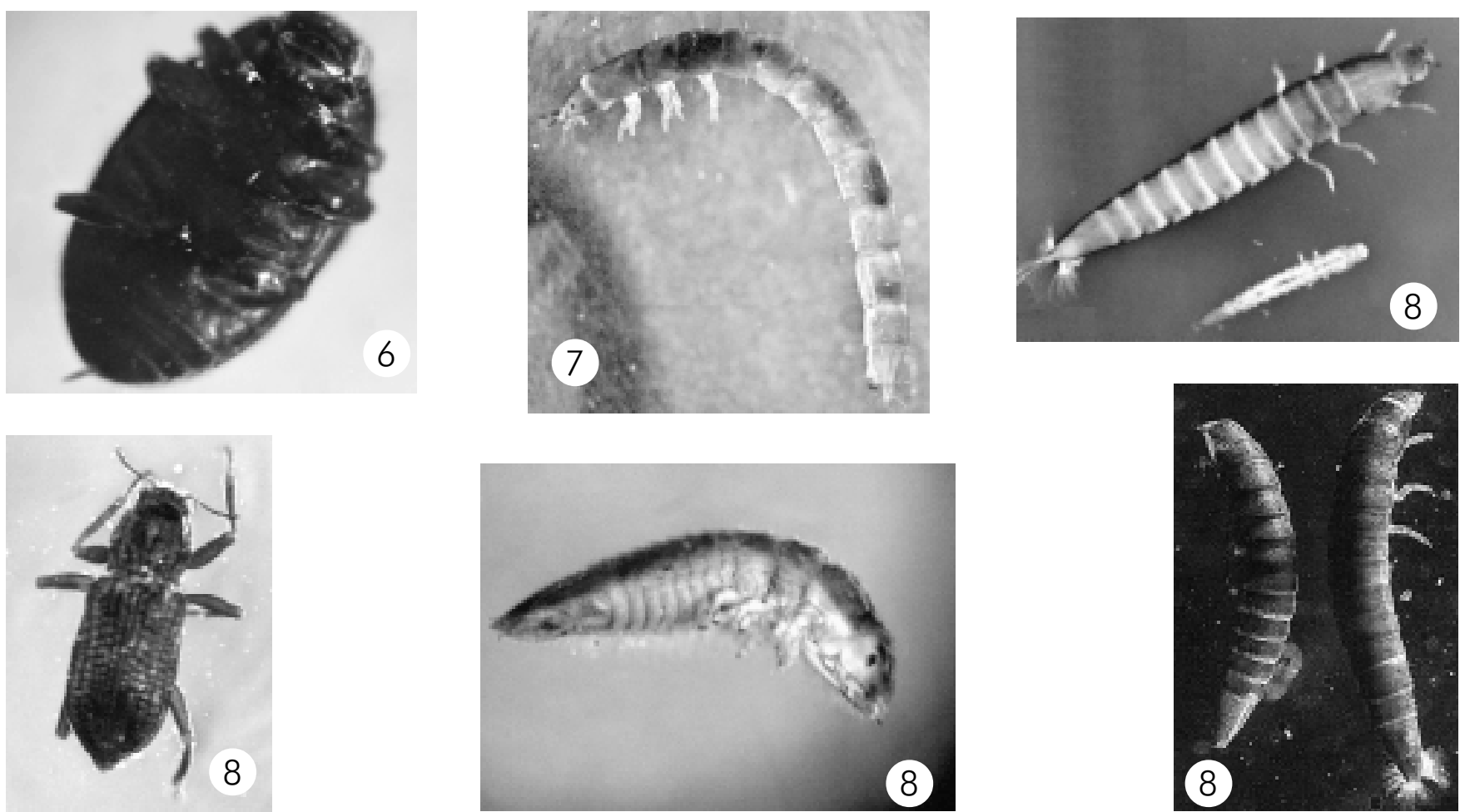


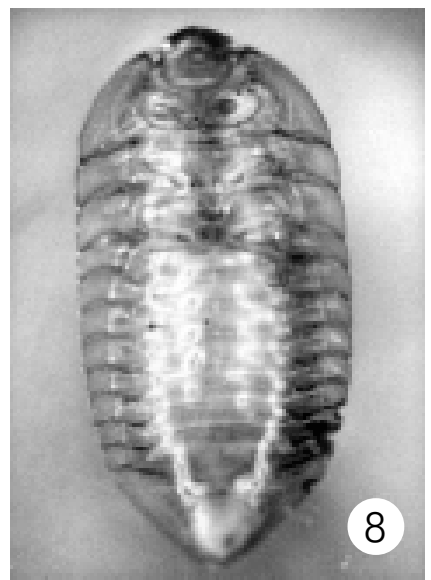

8
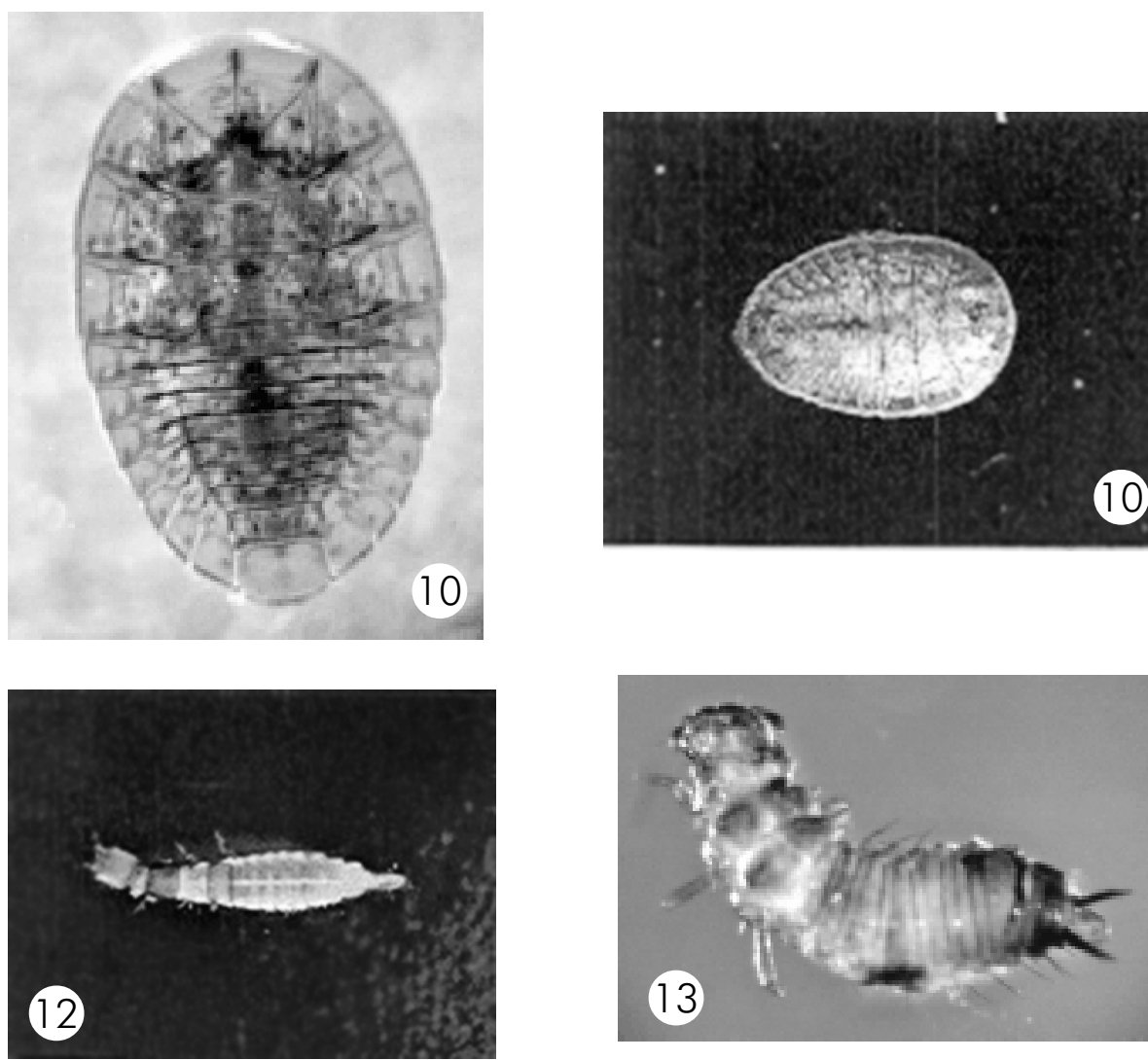
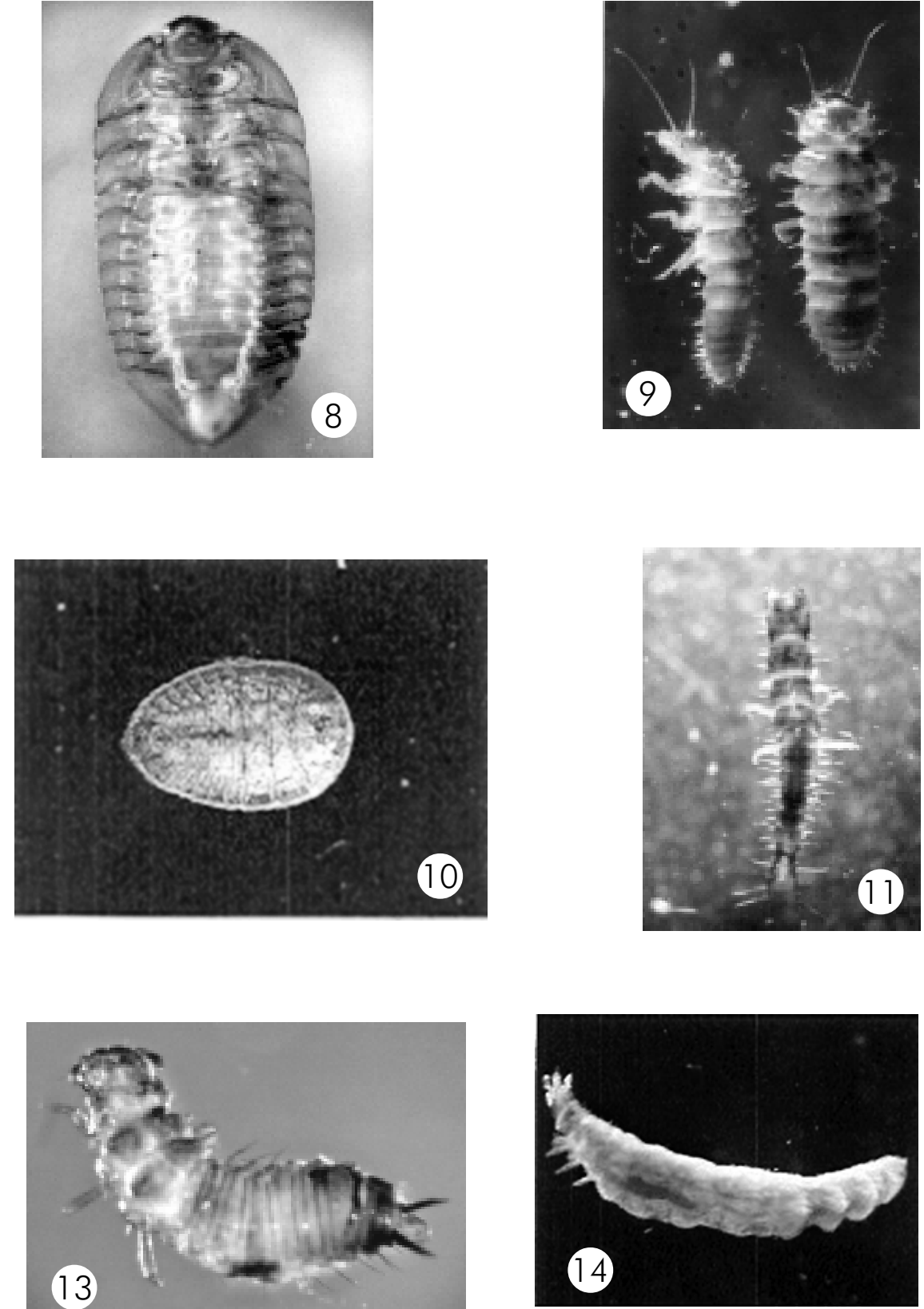
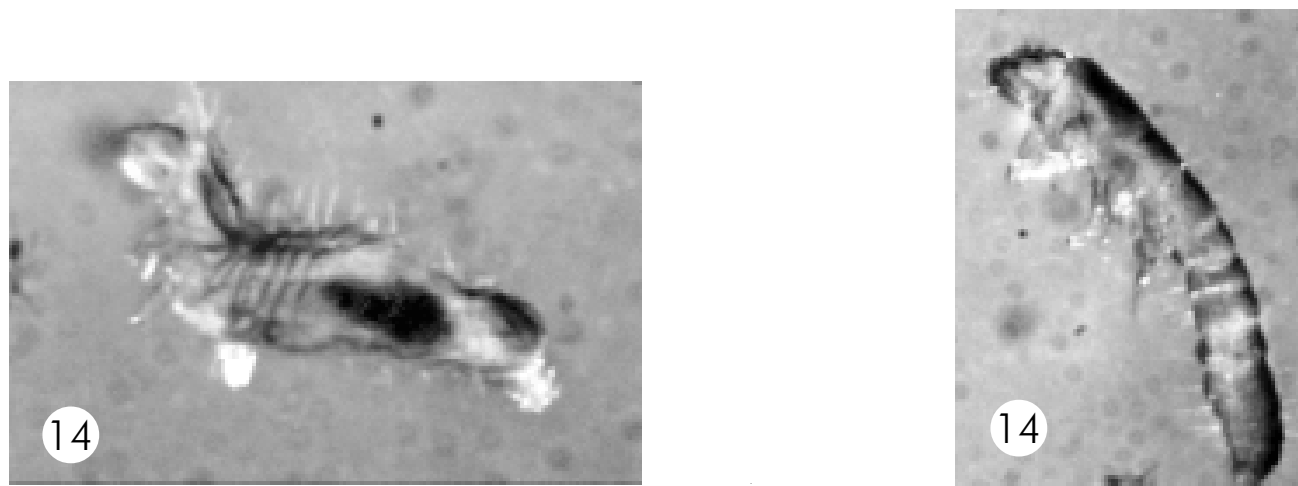
Macroinvertebrados del Norte de Santander

Orden: Collembola

\begin{tabular}{|c|c|c|c|c|c|c|c|c|c|}
\hline \multirow{2}{*}{\multicolumn{2}{|c|}{ FAMILIA }} & \multicolumn{2}{|c|}{ Cuenca río Zulia } & \multicolumn{2}{|c|}{$\begin{array}{l}\text { Cuenca río } \\
\text { Pamplonita }\end{array}$} & \multicolumn{2}{|c|}{$\begin{array}{l}\text { Cuenca río } \\
\text { Algodonal }\end{array}$} & \multicolumn{2}{|c|}{$\begin{array}{c}\text { Cuenca río } \\
\text { Táchira }\end{array}$} \\
\hline & & msnm & ${ }^{\circ} \mathrm{C}$ & msnm & ${ }^{\circ} \mathrm{C}$ & msnm & ${ }^{\circ} \mathrm{C}$ & msnm & ${ }^{\circ} \mathrm{C}$ \\
\hline 15 & Isotomidae & & & $\begin{array}{l}2900 \\
2800 \\
1045 \\
719 \\
250 \\
160\end{array}$ & $\begin{array}{l}11.5 \\
12.5 \\
19.5 \\
24 \\
33 \\
30\end{array}$ & & & & \\
\hline
\end{tabular}

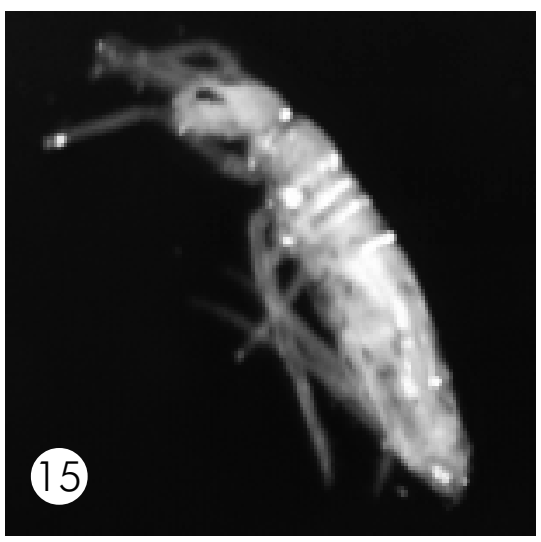

Orden: Diptera

\begin{tabular}{|c|c|c|c|c|c|c|c|c|c|}
\hline \multirow{2}{*}{\multicolumn{2}{|c|}{ FAMILIA }} & \multicolumn{2}{|c|}{ Cuenca río Zulia } & \multicolumn{2}{|c|}{$\begin{array}{l}\text { Cuenca río } \\
\text { Pamplonita }\end{array}$} & \multicolumn{2}{|c|}{$\begin{array}{l}\text { Cuenca río } \\
\text { Algodonal }\end{array}$} & \multicolumn{2}{|c|}{$\begin{array}{c}\text { Cuenca río } \\
\text { Táchira }\end{array}$} \\
\hline & & msnm & ${ }^{\circ} \mathrm{C}$ & msnm & ${ }^{\circ} \mathrm{C}$ & msnm & ${ }^{\circ} \mathrm{C}$ & msnm & ${ }^{\circ} \mathrm{C}$ \\
\hline 16 & Chironomidae & $\begin{array}{l}1298 \\
1200 \\
925 \\
904 \\
279\end{array}$ & $\begin{array}{l}18.7 \\
21.8 \\
23.2 \\
24 \\
24\end{array}$ & $\begin{array}{l}2900 \\
2800 \\
2400 \\
2314 \\
2249 \\
2120 \\
1045 \\
719 \\
400 \\
250 \\
200 \\
160 \\
100 \\
50\end{array}$ & $\begin{array}{l}11.5 \\
12.5 \\
14.5 \\
14.5 \\
14.5 \\
17.5 \\
19.5 \\
24 \\
25 \\
33 \\
34.5 \\
30 \\
32 \\
33.5\end{array}$ & $\begin{array}{l}1379 \\
1310 \\
1233 \\
1209 \\
1206 \\
970\end{array}$ & $\begin{array}{l}22 \\
20.2 \\
22 \\
24.8 \\
22 \\
23.5\end{array}$ & $\begin{array}{l}2435 \\
1800 \\
1200 \\
900 \\
300 \\
250\end{array}$ & $\begin{array}{l}25.1 \\
27.5 \\
26.5 \\
35.5 \\
30.2 \\
33\end{array}$ \\
\hline 17 & Ceratopogoniidae & 904 & 24 & $\begin{array}{l}2900 \\
2800 \\
2314 \\
2249 \\
719 \\
400 \\
250 \\
200 \\
160\end{array}$ & $\begin{array}{l}11.5 \\
12.5 \\
14.5 \\
14.5 \\
24 \\
25 \\
33 \\
34.5 \\
30\end{array}$ & 1233 & 22 & $\begin{array}{l}2435 \\
250\end{array}$ & $\begin{array}{c}25.1 \\
33\end{array}$ \\
\hline 18 & Blephariceridae & $\begin{array}{l}925 \\
904\end{array}$ & $\begin{array}{c}23.2 \\
24\end{array}$ & $\begin{array}{l}2800 \\
2314\end{array}$ & $\begin{array}{l}12.5 \\
14.5\end{array}$ & & & & \\
\hline 19 & Psychodidae & 925. & 23.2 & $\begin{array}{l}2400 \\
2314 \\
2249 \\
2120 \\
250 \\
200 \\
160\end{array}$ & $\begin{array}{l}14.5 \\
14.5 \\
14.5 \\
19.5 \\
33 \\
34.5 \\
30\end{array}$ & & & 1200 & 26.5 \\
\hline
\end{tabular}




\begin{tabular}{|c|c|c|c|c|c|c|c|c|c|}
\hline \multirow{2}{*}{\multicolumn{2}{|c|}{ FAMILIA }} & \multicolumn{2}{|c|}{ Cuenca río Zulia } & \multicolumn{2}{|c|}{$\begin{array}{l}\text { Cuenca río } \\
\text { Pamplonita }\end{array}$} & \multicolumn{2}{|c|}{$\begin{array}{l}\text { Cuenca río } \\
\text { Algodonal }\end{array}$} & \multicolumn{2}{|c|}{$\begin{array}{l}\text { Cuenca río } \\
\text { Táchira }\end{array}$} \\
\hline & & $\mathrm{msnm}$ & ${ }^{\circ} \mathrm{C}$ & $\mathrm{msnm}$ & ${ }^{\circ} \mathrm{C}$ & msnm & ${ }^{\circ} \mathrm{C}$ & $\mathrm{msnm}$ & ${ }^{\circ} \mathrm{C}$ \\
\hline 20 & Culicidae & & & $\begin{array}{l}2120 \\
200\end{array}$ & $\begin{array}{l}19.5 \\
345\end{array}$ & & & & \\
\hline 21 & Simuliidae & $\begin{array}{c}1298 \\
925 \\
904\end{array}$ & $\begin{array}{l}18.7 \\
23.2 \\
24\end{array}$ & $\begin{array}{l}2900 \\
2800 \\
2400 \\
2314 \\
2249 \\
2120 \\
1045 \\
719 \\
400 \\
250 \\
200 \\
160 \\
100 \\
50\end{array}$ & $\begin{array}{l}11.5 \\
12.5 \\
14.5 \\
14.5 \\
14.5 \\
17.5 \\
19.5 \\
24 \\
25 \\
33 \\
34.5 \\
30 \\
32 \\
33.5\end{array}$ & $\begin{array}{l}1379 \\
1392 \\
1310 \\
1233 \\
1206\end{array}$ & $\begin{array}{c}22 \\
21 \\
20.2 \\
22 \\
22\end{array}$ & $\begin{array}{l}2435 \\
900 \\
300 \\
250\end{array}$ & $\begin{array}{c}25.7 \\
35.5 \\
30.2 \\
33\end{array}$ \\
\hline 22 & Dixidae & 925 & 23.2 & $\begin{array}{l}719 \\
400\end{array}$ & $\begin{array}{l}24 \\
25\end{array}$ & & & & \\
\hline 23 & Muscidae & & & $\begin{array}{l}2400 \\
2314 \\
2249 \\
719 \\
200 \\
\end{array}$ & $\begin{array}{l}14.5 \\
14.5 \\
14.5 \\
24 \\
34.5\end{array}$ & & & & \\
\hline 24 & Empididae & 925 & 23.2 & $\begin{array}{l}2800 \\
2314 \\
2249 \\
1045 \\
400 \\
250 \\
100\end{array}$ & $\begin{array}{l}12.5 \\
14.5 \\
14.5 \\
19.5 \\
25 \\
33 \\
32\end{array}$ & 970 & 23.5 & 1200 & 26.5 \\
\hline 25 & Dolichopodidae & & & $\begin{array}{l}2400 \\
160\end{array}$ & $\begin{array}{l}74.5 \\
32\end{array}$ & & & & \\
\hline 26 & Tipulidae & 904 & 24 & $\begin{array}{l}2900 \\
2800 \\
2400 \\
2314 \\
2249 \\
2120\end{array}$ & $\begin{array}{l}11.5 \\
12.5 \\
14.5 \\
14.5 \\
14.5 \\
19.5\end{array}$ & 1310 & 20.2 & $\begin{array}{l}2435 \\
1800\end{array}$ & $\begin{array}{l}25.1 \\
27.6\end{array}$ \\
\hline 27 & Ephydridae & & & $\begin{array}{c}2314 \\
200 \\
160\end{array}$ & $\begin{array}{l}14.5 \\
34.5 \\
32\end{array}$ & & & & \\
\hline 28 & Syrphidae & & & $\begin{array}{l}2120 \\
250 \\
200\end{array}$ & $\begin{array}{l}19.5 \\
33 \\
34.5\end{array}$ & & & & \\
\hline 29 & Diptera & & & $\begin{array}{c}2800 \\
400 \\
200\end{array}$ & $\begin{array}{c}12.5 \\
25 \\
34.5\end{array}$ & & & & \\
\hline
\end{tabular}
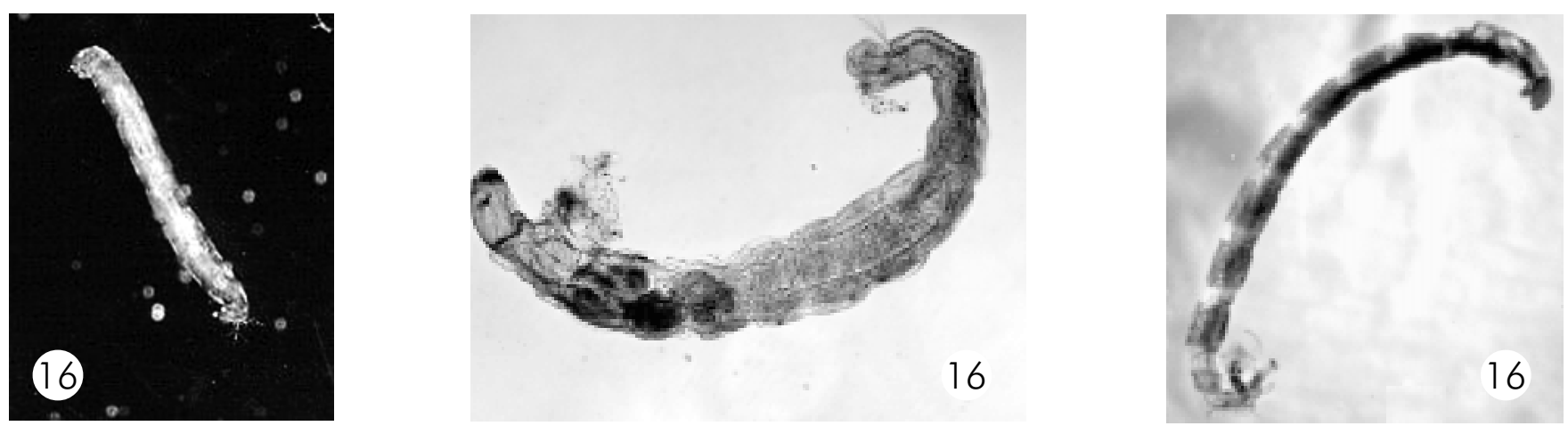

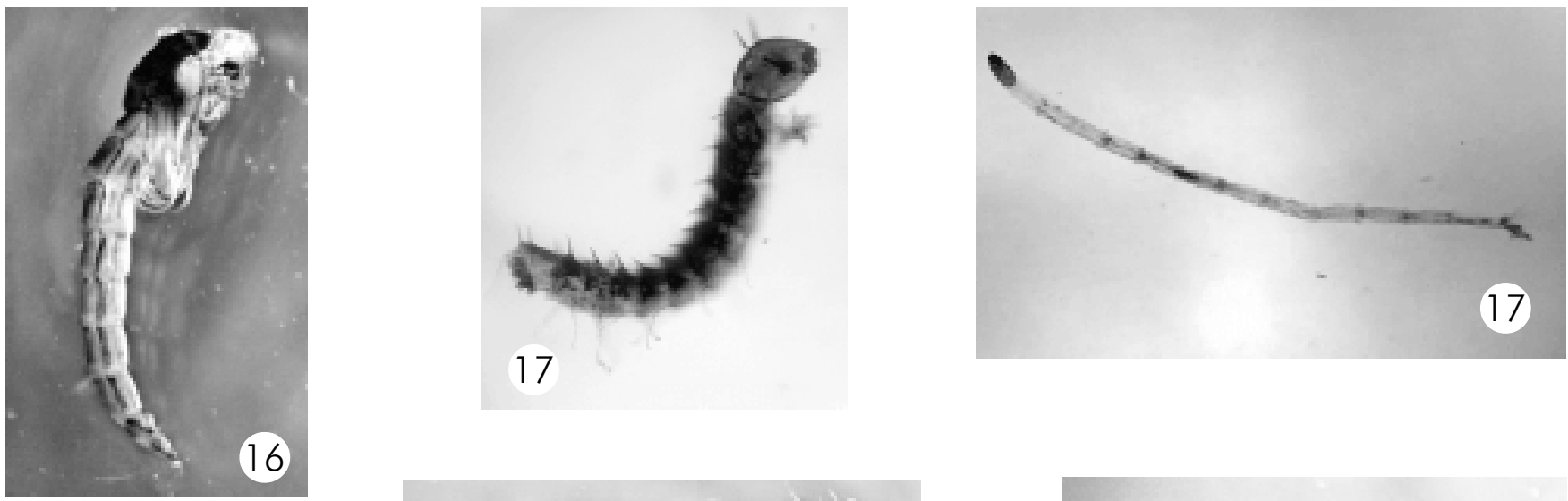

\section{7}
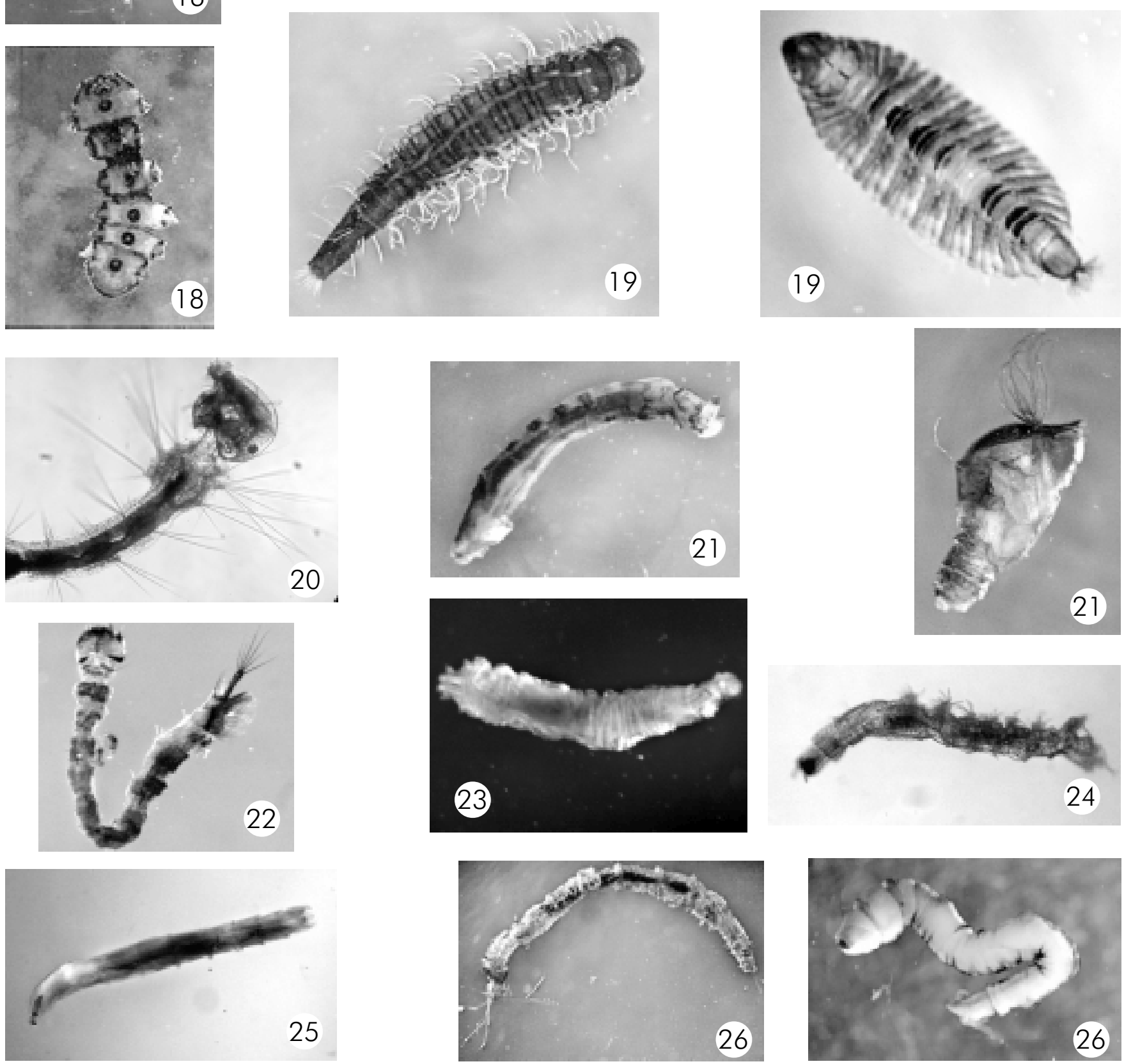

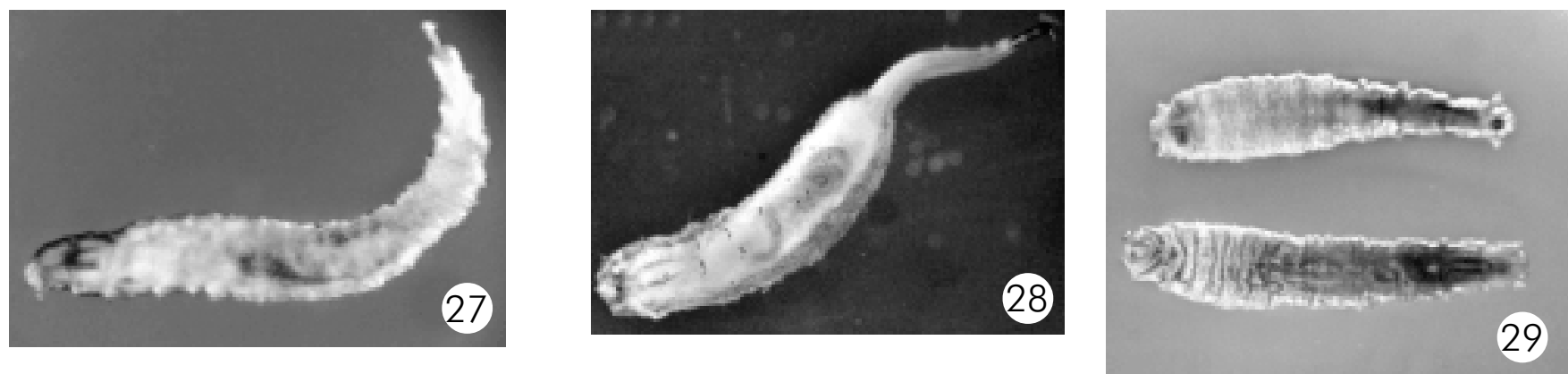

Orden: Ephemeroptera

\begin{tabular}{|c|c|c|c|c|c|c|c|c|c|}
\hline \multirow{2}{*}{\multicolumn{2}{|c|}{ FAMILIA }} & \multicolumn{2}{|c|}{ Cuenca río Zulia } & \multicolumn{2}{|c|}{$\begin{array}{l}\text { Cuenca río } \\
\text { Pamplonita }\end{array}$} & \multicolumn{2}{|c|}{$\begin{array}{l}\text { Cuenca río } \\
\text { Algodonal }\end{array}$} & \multicolumn{2}{|c|}{$\begin{array}{l}\text { Cuenca río } \\
\text { Táchira }\end{array}$} \\
\hline & & msnm & ${ }^{\circ} \mathrm{C}$ & msnm & ${ }^{\circ} \mathrm{C}$ & msnm & ${ }^{\circ} \mathrm{C}$ & msnm & ${ }^{\circ} \mathrm{C}$ \\
\hline 30 & Baetidae & $\begin{array}{l}1298 \\
1200 \\
925 \\
904 \\
279 \\
272 \\
50\end{array}$ & $\begin{array}{l}18.7 \\
21.8 \\
23.2 \\
24 \\
24 \\
23.7 \\
32\end{array}$ & $\begin{array}{l}2900 \\
2800 \\
2400 \\
2314 \\
2249 \\
2120 \\
1045 \\
719 \\
400 \\
250 \\
160 \\
100 \\
50\end{array}$ & $\begin{array}{l}11.5 \\
12.5 \\
14.5 \\
14.5 \\
14.5 \\
17.5 \\
19.5 \\
24 \\
25 \\
33 \\
30 \\
32 \\
33.5\end{array}$ & $\begin{array}{l}1379 \\
1392 \\
1233 \\
970\end{array}$ & $\begin{array}{c}22 \\
21 \\
22 \\
23.5\end{array}$ & $\begin{array}{l}2435 \\
1800 \\
1200 \\
900 \\
300 \\
250\end{array}$ & $\begin{array}{c}25.1 \\
27.6 \\
26.5 \\
35.5 \\
30.2 \\
33\end{array}$ \\
\hline 31 & Leptohyphidae & $\begin{array}{l}1298 \\
1200 \\
925 \\
904 \\
279 \\
272 \\
50\end{array}$ & $\begin{array}{l}18.7 \\
21.8 \\
23.2 \\
24 \\
24 \\
23.7 \\
32\end{array}$ & $\begin{array}{l}1045 \\
719 \\
400 \\
250 \\
160 \\
100 \\
50\end{array}$ & $\begin{array}{l}19.5 \\
24 \\
25 \\
33 \\
30 \\
32 \\
33.5\end{array}$ & $\begin{array}{l}1379 \\
1392 \\
1310 \\
1233 \\
1209 \\
1206 \\
970\end{array}$ & $\begin{array}{c}22 \\
21 \\
20.2 \\
22 \\
24.8 \\
22 \\
23.5\end{array}$ & $\begin{array}{l}1800 \\
1200 \\
900 \\
300 \\
250\end{array}$ & $\begin{array}{l}27.6 \\
26.5 \\
35.5 \\
30.2 \\
33\end{array}$ \\
\hline 32 & Oligoneuriidae & 279 & 24 & $\begin{array}{l}2900 \\
1045 \\
719 \\
400 \\
250 \\
50\end{array}$ & $\begin{array}{l}11.5 \\
19.5 \\
24 \\
25 \\
33 \\
33.5\end{array}$ & $\begin{array}{l}1392 \\
1310 \\
1233 \\
1209 \\
1206\end{array}$ & $\begin{array}{c}21 \\
20.2 \\
22 \\
24.8 \\
22\end{array}$ & & \\
\hline 33 & Leptophlebiidae & $\begin{array}{l}1298 \\
1200 \\
925 \\
904 \\
279 \\
272 \\
50\end{array}$ & $\begin{array}{l}18.7 \\
21.8 \\
23.2 \\
24 \\
24 \\
23.7 \\
32\end{array}$ & $\begin{array}{l}2800 \\
1045 \\
719 \\
400 \\
250 \\
160 \\
100 \\
50\end{array}$ & $\begin{array}{l}12.5 \\
19.5 \\
24 \\
25 \\
33 \\
30 \\
32 \\
33.5\end{array}$ & $\begin{array}{l}1379 \\
1392 \\
1233 \\
1206 \\
970\end{array}$ & $\begin{array}{c}22 \\
21 \\
22 \\
22 \\
23.5\end{array}$ & $\begin{array}{l}2435 \\
1800 \\
1200 \\
900 \\
300 \\
250\end{array}$ & $\begin{array}{l}25.7 \\
27.6 \\
26.5 \\
35.5 \\
30.2 \\
33\end{array}$ \\
\hline
\end{tabular}
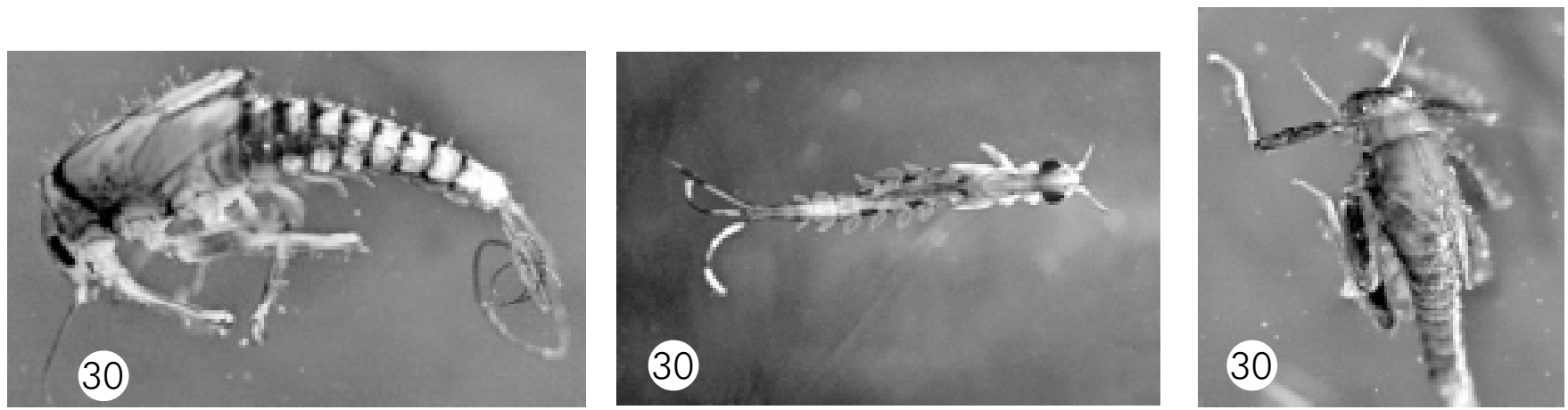

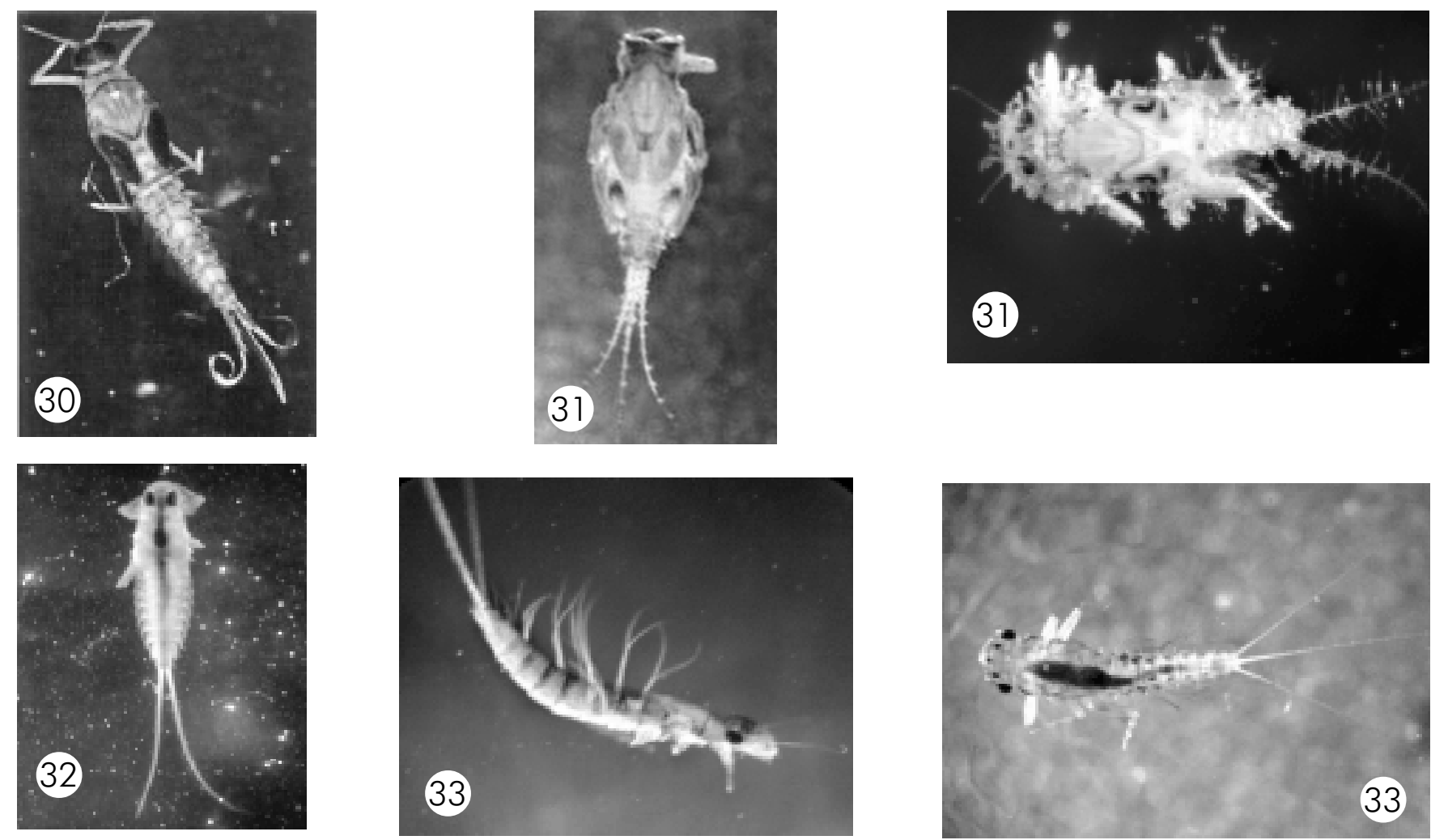

Orden: Hemiptera

\begin{tabular}{|c|c|c|c|c|c|c|c|c|c|}
\hline \multirow{2}{*}{\multicolumn{2}{|c|}{ FAMILIA }} & \multicolumn{2}{|c|}{ Cuenca río Zulia } & \multicolumn{2}{|c|}{$\begin{array}{l}\text { Cuenca río } \\
\text { Pamplonita }\end{array}$} & \multicolumn{2}{|c|}{$\begin{array}{l}\text { Cuenca río } \\
\text { Algodonal }\end{array}$} & \multicolumn{2}{|c|}{$\begin{array}{c}\text { Cuenca río } \\
\text { Táchira }\end{array}$} \\
\hline & & msnm & ${ }^{\circ} \mathrm{C}$ & msnm & ${ }^{\circ} \mathrm{C}$ & msnm & ${ }^{\circ} \mathrm{C}$ & msnm & ${ }^{\circ} \mathrm{C}$ \\
\hline 34 & Hebridae & & & 400 & 25 & & & & \\
\hline 35 & Veliidae & $\begin{array}{l}925 \\
279 \\
272\end{array}$ & $\begin{array}{l}23.2 \\
24 \\
23.7\end{array}$ & $\begin{array}{l}719 \\
400 \\
50\end{array}$ & $\begin{array}{c}24 \\
25 \\
33.5\end{array}$ & $\begin{array}{l}1379 \\
1392 \\
1310 \\
1209 \\
1206\end{array}$ & $\begin{array}{c}22 \\
21 \\
20.2 \\
24.8 \\
22\end{array}$ & 1800 & 27.6 \\
\hline 36 & Naucoridae & $\begin{array}{l}279 \\
272\end{array}$ & $\begin{array}{c}24 \\
23.7\end{array}$ & $\begin{array}{l}1045 \\
719 \\
400 \\
250 \\
160\end{array}$ & $\begin{array}{l}19.5 \\
24 \\
25 \\
33 \\
30\end{array}$ & $\begin{array}{l}1310 \\
1233\end{array}$ & $\begin{array}{l}20.2 \\
22\end{array}$ & 900 & 35.5 \\
\hline 37 & Gerridae & & & $\begin{array}{l}719 \\
50\end{array}$ & $\begin{array}{c}24 \\
33.5\end{array}$ & & & & \\
\hline 38 & Gelastocoridae & & & 250 & 33 & & & & \\
\hline
\end{tabular}

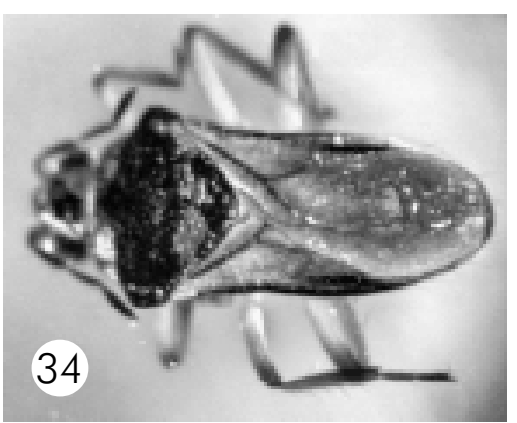

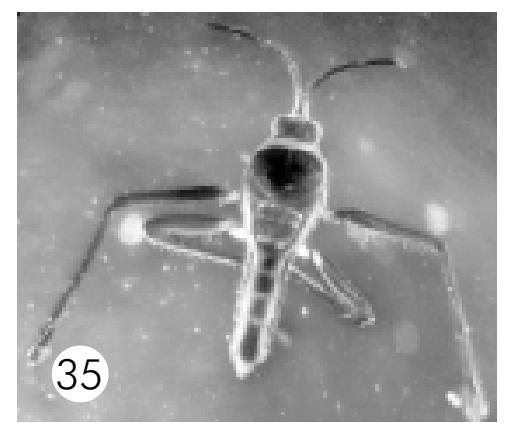

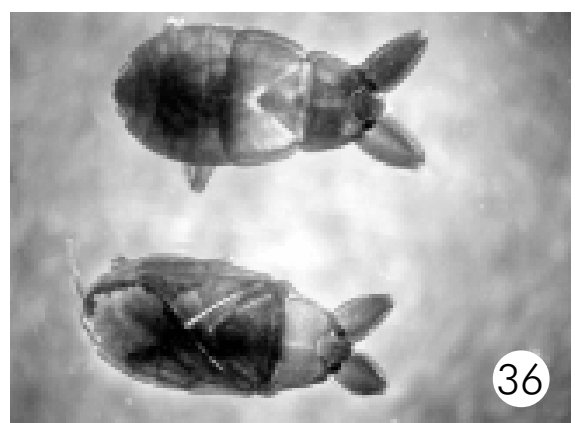

Revista Respuestas - Universidad Francisco de Paula Santander Año 10 No. 1 
Macroinvertebrados del Norte de Santander
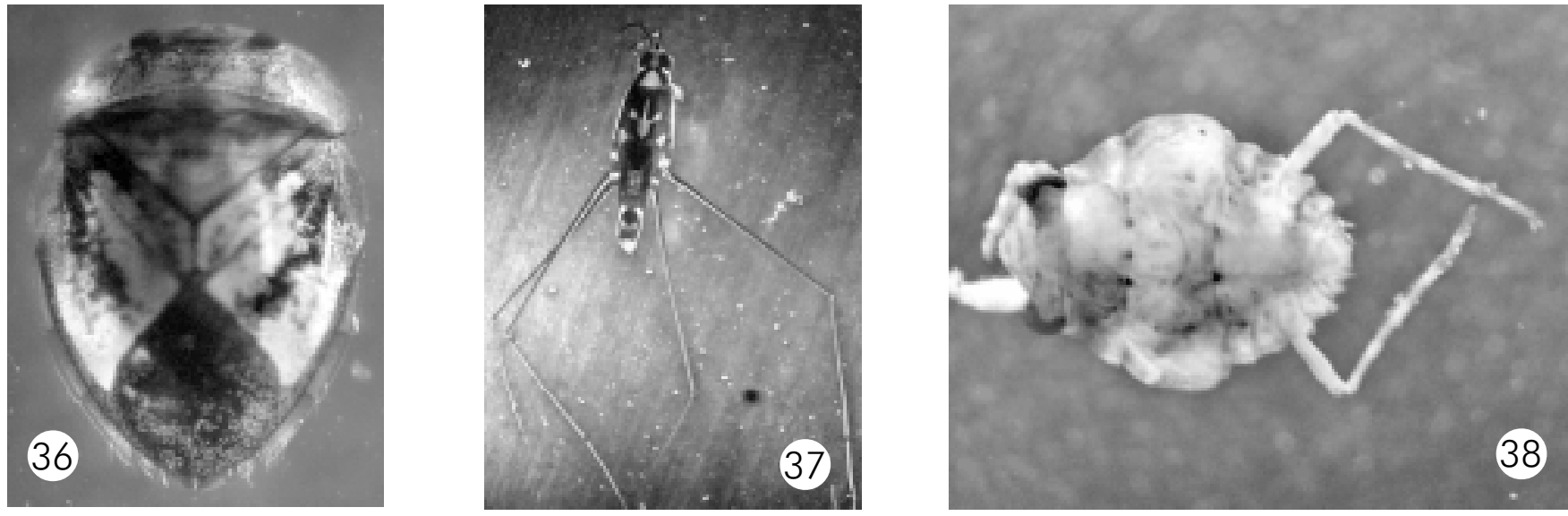

Orden: lepidoptera

\begin{tabular}{|c|c|c|c|c|c|c|c|c|c|}
\hline \multirow{2}{*}{\multicolumn{2}{|c|}{ FAMILIA }} & \multicolumn{2}{|c|}{ Cuenca río Zulia } & \multicolumn{2}{|c|}{$\begin{array}{l}\text { Cuenca río } \\
\text { Pamplonita }\end{array}$} & \multicolumn{2}{|c|}{$\begin{array}{l}\text { Cuenca río } \\
\text { Algodonal }\end{array}$} & \multicolumn{2}{|c|}{$\begin{array}{c}\text { Cuenca río } \\
\text { Táchira }\end{array}$} \\
\hline & & msnm & ${ }^{\circ} \mathrm{C}$ & msnm & ${ }^{\circ} \mathrm{C}$ & msnm & ${ }^{\circ} \mathrm{C}$ & msnm & ${ }^{\circ} \mathrm{C}$ \\
\hline 39 & Pyralidae & 1298 & 18.7 & $\begin{array}{c}1045 \\
50\end{array}$ & $\begin{array}{l}19.5 \\
33.5\end{array}$ & $\begin{array}{l}1233 \\
970\end{array}$ & $\begin{array}{c}22 \\
23.5\end{array}$ & & \\
\hline 40 & Cossidae cf & & & 2800 & 12.5 & & & & \\
\hline
\end{tabular}
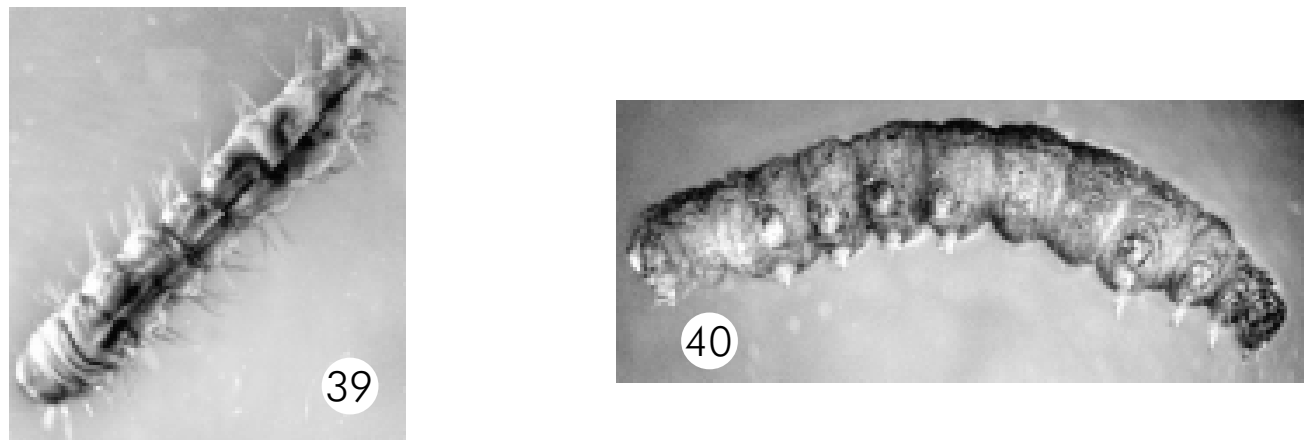

Orden: Megaloptera

\begin{tabular}{|l|l|c|c|c|c|c|c|c|c|}
\hline \multirow{2}{*}{ FAMILIA } & \multicolumn{2}{|c|}{ Cuenca río Zulia } & \multicolumn{2}{c|}{$\begin{array}{c}\text { Cuenca río } \\
\text { Pamplonita }\end{array}$} & \multicolumn{2}{c|}{$\begin{array}{c}\text { Cuenca río } \\
\text { Algodonal }\end{array}$} & \multicolumn{2}{c|}{$\begin{array}{c}\text { Cuenca río } \\
\text { Táchira }\end{array}$} \\
\cline { 3 - 10 } \multicolumn{2}{|l|}{} & msnm & ${ }^{\circ} \mathrm{C}$ & $\mathrm{msnm}$ & ${ }^{\circ} \mathrm{C}$ & $\mathrm{msnm}$ & ${ }^{\circ} \mathrm{C}$ & $\mathrm{msnm}$ & ${ }^{\circ} \mathrm{C}$ \\
\hline \multirow{3}{*}{41} & Corydalidae & 1200 & 21.8 & 1045 & 19.5 & 1379 & 22 & 1800 & 27.6 \\
& 272 & 23.7 & 719 & 24 & 1392 & 21 & 1200 & 26.5 \\
& & & 400 & 25 & 1310 & 20.2 & 900 & 35.5 \\
& & & 250 & 33 & 1209 & 24.8 & & \\
& & & 100 & 32.5 & 970 & 23.5 & & \\
\hline
\end{tabular}
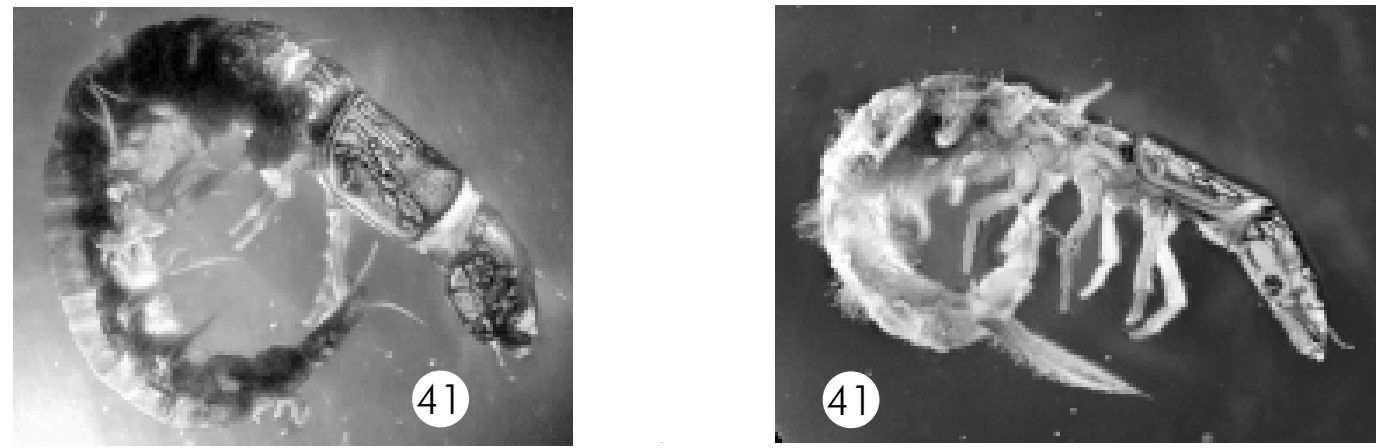
Macroinvertebrados del Norte de Santander

Orden: Odonata

\begin{tabular}{|c|c|c|c|c|c|c|c|c|c|}
\hline \multirow{2}{*}{\multicolumn{2}{|c|}{ FAMILIA }} & \multicolumn{2}{|c|}{ Cuenca río Zulia } & \multicolumn{2}{|c|}{$\begin{array}{l}\text { Cuenca río } \\
\text { Pamplonita }\end{array}$} & \multicolumn{2}{|c|}{$\begin{array}{l}\text { Cuenca río } \\
\text { Algodonal }\end{array}$} & \multicolumn{2}{|c|}{$\begin{array}{l}\text { Cuenca río } \\
\text { Táchira }\end{array}$} \\
\hline & & msnm & ${ }^{\circ} \mathrm{C}$ & $\mathrm{msnm}$ & ${ }^{\circ} \mathrm{C}$ & msnm & ${ }^{\circ} \mathrm{C}$ & msnm & ${ }^{\circ} \mathrm{C}$ \\
\hline 42 & Calopterygidae & & & $\begin{array}{l}1045 \\
719 \\
400 \\
100 \\
50\end{array}$ & $\begin{array}{l}19.5 \\
24 \\
25 \\
32.5 \\
33.5\end{array}$ & $\begin{array}{l}1392 \\
1310 \\
1209 \\
1206 \\
970\end{array}$ & $\begin{array}{c}21 \\
20.2 \\
24.8 \\
22 \\
23.5\end{array}$ & & \\
\hline 43 & Libellulidae & 904 & 24 & $\begin{array}{l}2120 \\
719 \\
400 \\
250 \\
50\end{array}$ & $\begin{array}{l}17.5 \\
24 \\
25 \\
33 \\
33.5\end{array}$ & $\begin{array}{l}1209 \\
1206\end{array}$ & $\begin{array}{l}24.8 \\
22\end{array}$ & $\begin{array}{l}2435 \\
900 \\
300 \\
250\end{array}$ & $\begin{array}{c}25.1 \\
35.5 \\
30.2 \\
33\end{array}$ \\
\hline 44 & Coenagrionidae & 50 & 32 & $\begin{array}{l}250 \\
100 \\
50\end{array}$ & $\begin{array}{c}33 \\
32.5 \\
33.5\end{array}$ & 970 & 23.5 & 300 & 30.2 \\
\hline 45 & Aeshnidae & & & 2249 & 14.5 & & & & \\
\hline
\end{tabular}

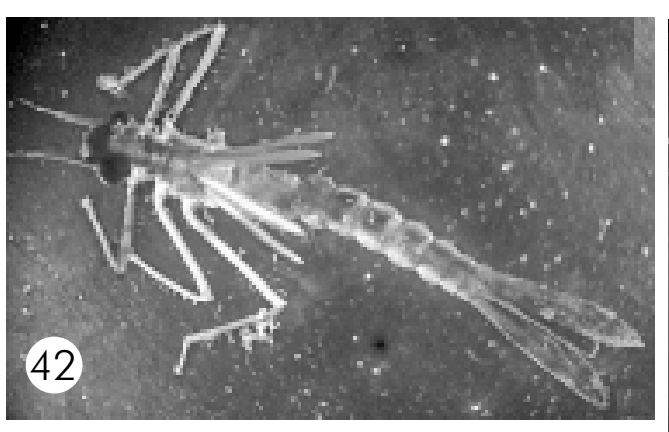

43

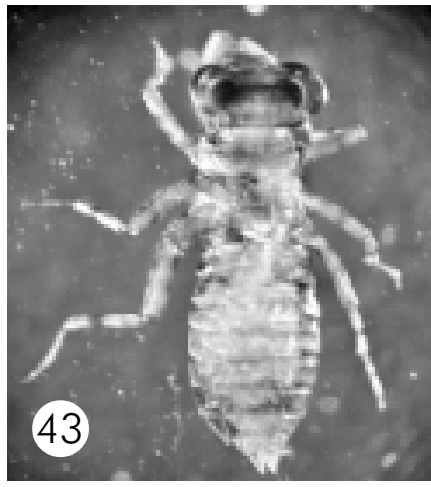

Orden: Plecoptera
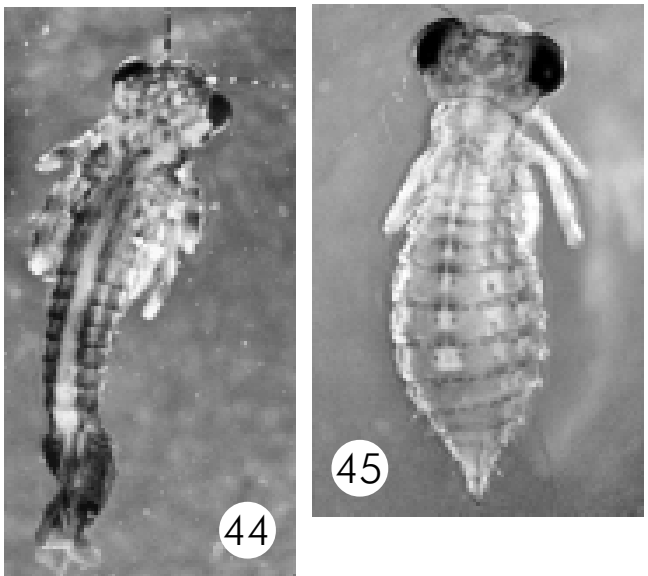

\begin{tabular}{|l|l|c|c|c|c|c|c|c|c|}
\hline \multirow{2}{*}{ FAMILIA } & \multicolumn{2}{|c|}{ Cuenca río Zulia } & \multicolumn{2}{c|}{$\begin{array}{c}\text { Cuenca río } \\
\text { Pamplonita }\end{array}$} & \multicolumn{2}{c|}{$\begin{array}{c}\text { Cuenca río } \\
\text { Algodonal }\end{array}$} & \multicolumn{2}{c|}{$\begin{array}{c}\text { Cuenca río } \\
\text { Táchira }\end{array}$} \\
\cline { 3 - 9 } \multicolumn{2}{|l|}{} & msnm & ${ }^{\circ} \mathrm{C}$ & $\mathrm{msnm}$ & ${ }^{\circ} \mathrm{C}$ & $\mathrm{msnm}$ & ${ }^{\circ} \mathrm{C}$ & $\mathrm{msnm}$ & ${ }^{\circ} \mathrm{C}$ \\
\hline \multirow{3}{*}{46} & Perlidae & 1200 & 21.8 & 2900 & 11.5 & 1379 & 22 & 2435 & 25.1 \\
& 925 & 23.2 & 2800 & 12.5 & 1392 & 21 & 1800 & 27.6 \\
& 904 & 24 & 2400 & 14.5 & 1233 & 22 & 1200 & 26.5 \\
& 279 & 24 & 1045 & 19.5 & & & 900 & 35.5 \\
& 272 & 23.7 & 719 & 24 & & & 250 & 33 \\
& & & 400 & 25 & & & & \\
& & & 250 & 33 & & & & \\
& & & 160 & 30 & & & & \\
& & & 100 & 32.5 & & & & \\
& & & & & & & & &
\end{tabular}

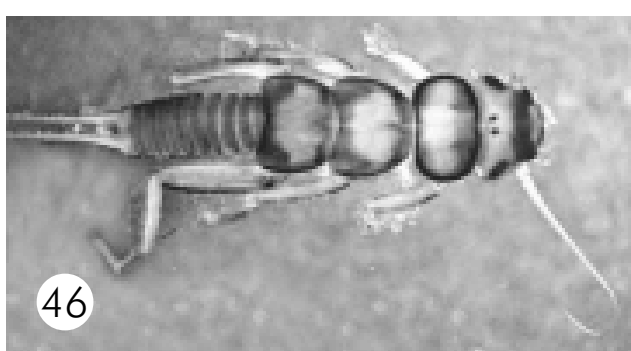

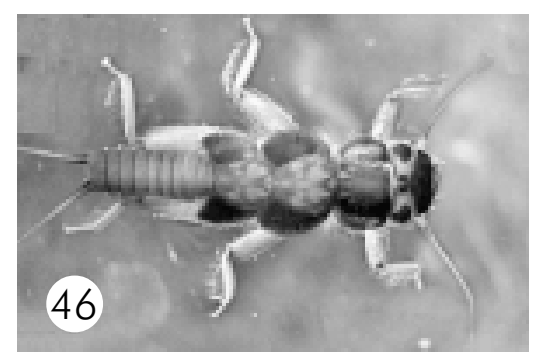

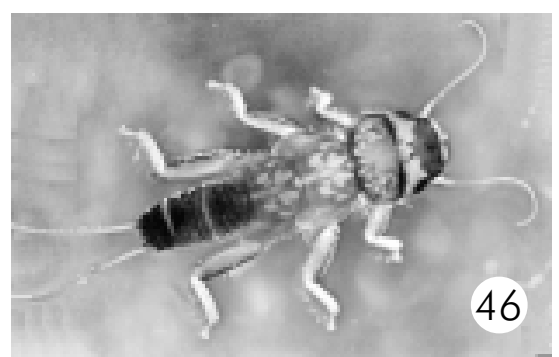

Revista Respuestas - Universidad Francisco de Paula Santander Año 10 No. 1 
Orden: Rhynchobdellida

\begin{tabular}{|l|l|c|c|c|c|c|c|c|c|}
\hline \multicolumn{2}{|l|}{ FAMILIA } & \multicolumn{2}{c|}{ Cuenca río Zulia } & \multicolumn{2}{c|}{$\begin{array}{c}\text { Cuenca río } \\
\text { Pamplonita }\end{array}$} & \multicolumn{2}{c|}{$\begin{array}{c}\text { Cuenca río } \\
\text { Algodonal }\end{array}$} & \multicolumn{2}{c|}{$\begin{array}{c}\text { Cuenca río } \\
\text { Táchira }\end{array}$} \\
\cline { 3 - 9 } \multicolumn{2}{|l}{} & msnm & ${ }^{\circ} \mathrm{C}$ & $\mathrm{msnm}$ & ${ }^{\circ} \mathrm{C}$ & $\mathrm{msnm}$ & ${ }^{\circ} \mathrm{C}$ & $\mathrm{msnm}$ & ${ }^{\circ} \mathrm{C}$ \\
\hline 47 & Glossiphoniidae & & & 2249 & 14.5 & 1310 & 20.2 & & \\
& & & & 2120 & 17.5 & 970 & 23.5 & \\
& & & & 1045 & 19.5 & & & & \\
& & & 250 & 33 & & & & \\
\hline 48 & Hirudinea & 272 & 23.7 & & & & & & \\
\hline
\end{tabular}
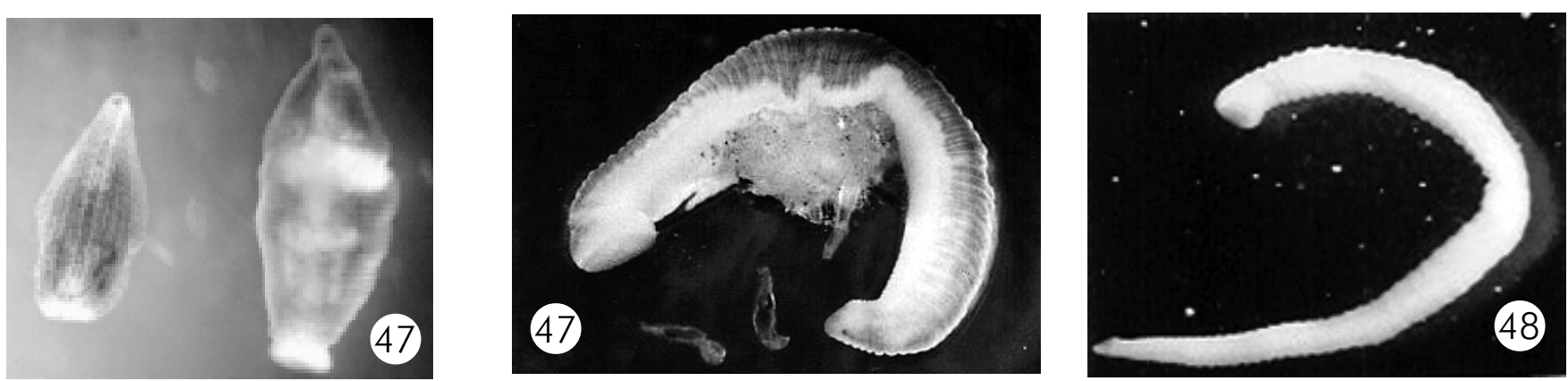

Orden: Trichoptera

\begin{tabular}{|c|c|c|c|c|c|c|c|c|c|}
\hline \multirow{2}{*}{\multicolumn{2}{|c|}{ FAMILIA }} & \multicolumn{2}{|c|}{ Cuenca río Zulia } & \multicolumn{2}{|c|}{$\begin{array}{l}\text { Cuenca río } \\
\text { Pamplonita }\end{array}$} & \multicolumn{2}{|c|}{$\begin{array}{l}\text { Cuenca río } \\
\text { Algodonal }\end{array}$} & \multicolumn{2}{|c|}{$\begin{array}{c}\text { Cuenca río } \\
\text { Táchira }\end{array}$} \\
\hline & & msnm & ${ }^{\circ} \mathrm{C}$ & $\mathrm{msnm}$ & ${ }^{\circ} \mathrm{C}$ & $\mathrm{msnm}$ & ${ }^{\circ} \mathrm{C}$ & $\mathrm{msnm}$ & ${ }^{\circ} \mathrm{C}$ \\
\hline 49 & Glossosomatidae & $\begin{array}{l}1298 \\
1200 \\
925 \\
279 \\
272 \\
50\end{array}$ & $\begin{array}{l}18.7 \\
21.8 \\
23.2 \\
24 \\
23.7 \\
32\end{array}$ & $\begin{array}{l}2900 \\
2800 \\
2400 \\
719 \\
400\end{array}$ & $\begin{array}{l}11.5 \\
12.5 \\
14.5 \\
24 \\
25\end{array}$ & $\begin{array}{l}1379 \\
1310 \\
1233\end{array}$ & $\begin{array}{c}22 \\
20.2 \\
22\end{array}$ & 2435 & 25.1 \\
\hline 50 & Hydrobiosidae & $\begin{array}{l}1298 \\
925 \\
904\end{array}$ & $\begin{array}{l}18.7 \\
23.2 \\
24\end{array}$ & $\begin{array}{l}2900 \\
2800 \\
2314 \\
2249 \\
1045 \\
719 \\
400 \\
250\end{array}$ & $\begin{array}{l}11.5 \\
12.5 \\
14.5 \\
14.5 \\
19.5 \\
24 \\
25 \\
33\end{array}$ & $\begin{array}{l}1379 \\
1392\end{array}$ & $\begin{array}{l}22 \\
21\end{array}$ & $\begin{array}{l}2435 \\
1800 \\
1200\end{array}$ & $\begin{array}{l}25.1 \\
27.6 \\
35.5\end{array}$ \\
\hline 51 & Hydropsychidae & $\begin{array}{l}1298 \\
1200 \\
925 \\
904 \\
279 \\
272 \\
50\end{array}$ & $\begin{array}{c}18.7 \\
21.8 \\
23.2 \\
24 \\
24 \\
23.7 \\
32\end{array}$ & $\begin{array}{c}2900 \\
2800 \\
2400 \\
2314 \\
1045 \\
719 \\
400 \\
250 \\
200 \\
160 \\
100 \\
50\end{array}$ & $\begin{array}{l}11.5 \\
12.5 \\
14.5 \\
14.5 \\
19.5 \\
24 \\
25 \\
33 \\
34.5 \\
30 \\
32.5 \\
33.5\end{array}$ & $\begin{array}{l}1379 \\
1392 \\
1310 \\
1233 \\
1209 \\
1206 \\
970\end{array}$ & $\begin{array}{c}22 \\
21 \\
20.2 \\
22 \\
24.8 \\
22 \\
23.5\end{array}$ & $\begin{array}{l}2435 \\
1800 \\
1200 \\
900\end{array}$ & $\begin{array}{l}25.1 \\
27.6 \\
26.5 \\
35.5\end{array}$ \\
\hline 52 & Leptoceridae & $\begin{array}{l}1298 \\
1200 \\
925 \\
904 \\
272\end{array}$ & $\begin{array}{l}18.7 \\
21.8 \\
23.2 \\
24 \\
23.7\end{array}$ & $\begin{array}{c}2900 \\
2800 \\
2314 \\
400 \\
50\end{array}$ & $\begin{array}{c}11.5 \\
12.5 \\
14.5 \\
25 \\
33.5\end{array}$ & $\begin{array}{l}1379 \\
1206\end{array}$ & $\begin{array}{l}22 \\
22\end{array}$ & & \\
\hline 53 & Philopotamidae & 50 & 32 & $\begin{array}{l}2314 \\
1045 \\
719 \\
400\end{array}$ & $\begin{array}{l}14.5 \\
19.5 \\
24 \\
25\end{array}$ & 1233 & 22 & & \\
\hline
\end{tabular}


Macroinvertebrados del Norte de Santander

\begin{tabular}{|c|c|c|c|c|c|c|c|c|c|}
\hline \multirow{2}{*}{\multicolumn{2}{|c|}{ FAMILIA }} & \multicolumn{2}{|c|}{ Cuenca río Zulia } & \multicolumn{2}{|c|}{$\begin{array}{l}\text { Cuenca río } \\
\text { Pamplonita }\end{array}$} & \multicolumn{2}{|c|}{$\begin{array}{l}\text { Cuenca río } \\
\text { Algodonal }\end{array}$} & \multicolumn{2}{|c|}{$\begin{array}{c}\text { Cuenca río } \\
\text { Táchira }\end{array}$} \\
\hline & & msnm & ${ }^{\circ} \mathrm{C}$ & msnm & ${ }^{\circ} \mathrm{C}$ & msnm & ${ }^{\circ} \mathrm{C}$ & msnm & ${ }^{\circ} \mathrm{C}$ \\
\hline 53 & Philopotamidae & 50 & 32 & $\begin{array}{l}2314 \\
1045 \\
719 \\
400\end{array}$ & $\begin{array}{l}14.5 \\
19.5 \\
24 \\
25\end{array}$ & 1233 & 22 & & \\
\hline 54 & Helicopsychidae & $\begin{array}{l}1298 \\
1200 \\
279 \\
272\end{array}$ & $\begin{array}{l}18.7 \\
21.8 \\
24 \\
23.7\end{array}$ & $\begin{array}{l}2900 \\
719\end{array}$ & $\begin{array}{l}11.5 \\
24\end{array}$ & & & 1200 & 26.5 \\
\hline 55 & Hydroptilidae & $\begin{array}{l}1200 \\
925 \\
50\end{array}$ & $\begin{array}{l}21.8 \\
23.2 \\
32\end{array}$ & $\begin{array}{l}2900 \\
2800 \\
2314 \\
719 \\
100 \\
50\end{array}$ & $\begin{array}{l}11.5 \\
12.5 \\
14.5 \\
24 \\
32.5 \\
33.5\end{array}$ & & & 250 & 33 \\
\hline 56 & Odontoceridae & & & $\begin{array}{l}2900 \\
2800 \\
2314 \\
\end{array}$ & $\begin{array}{l}11.5 \\
12.5 \\
14.5\end{array}$ & & & & \\
\hline 57 & Xiphocentronidae & & & $\begin{array}{l}2900 \\
2800 \\
2314 \\
719 \\
400 \\
\end{array}$ & $\begin{array}{l}11.5 \\
12.5 \\
14.5 \\
24 \\
25 \\
\end{array}$ & & & & \\
\hline 58 & Calamoceratidae & & & $\begin{array}{l}2900 \\
2800\end{array}$ & $\begin{array}{l}11.5 \\
12.5\end{array}$ & 1233 & 22 & & \\
\hline 59 & Polycentropodidae & 904 & 24 & 2120 & 17.5 & & & & \\
\hline
\end{tabular}
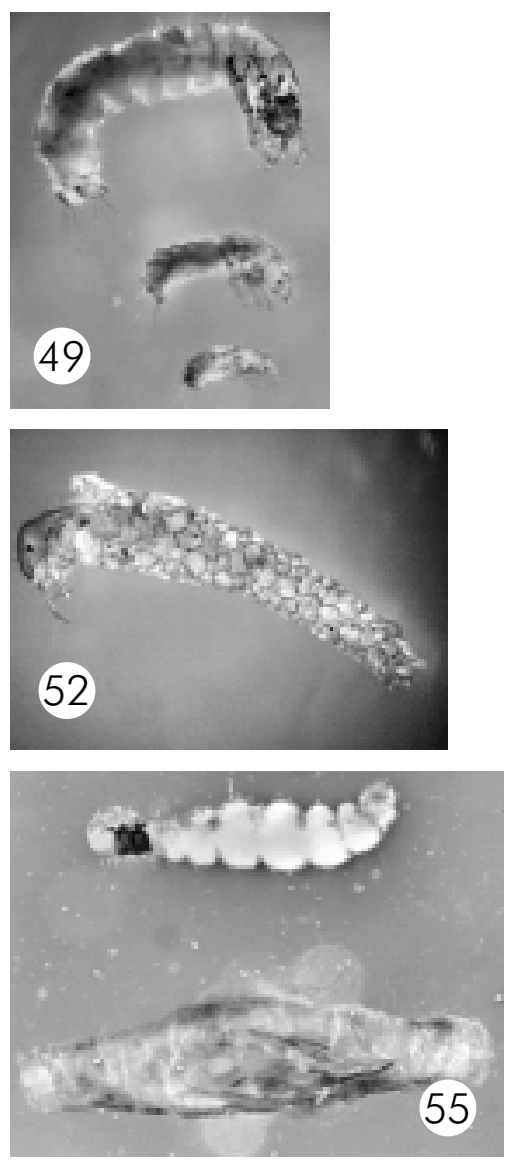
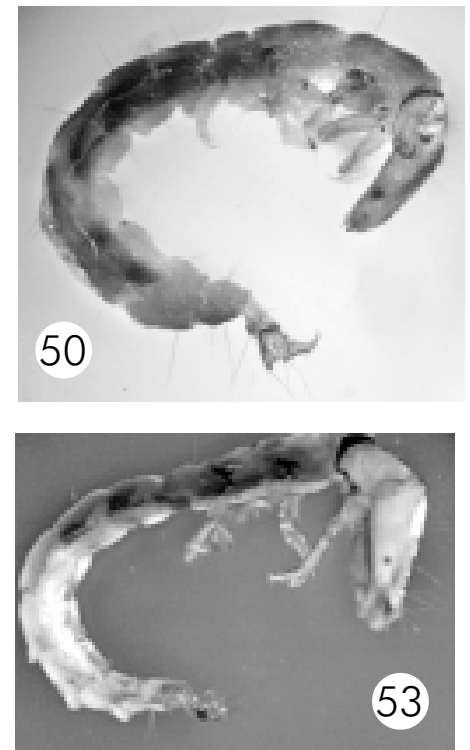

51
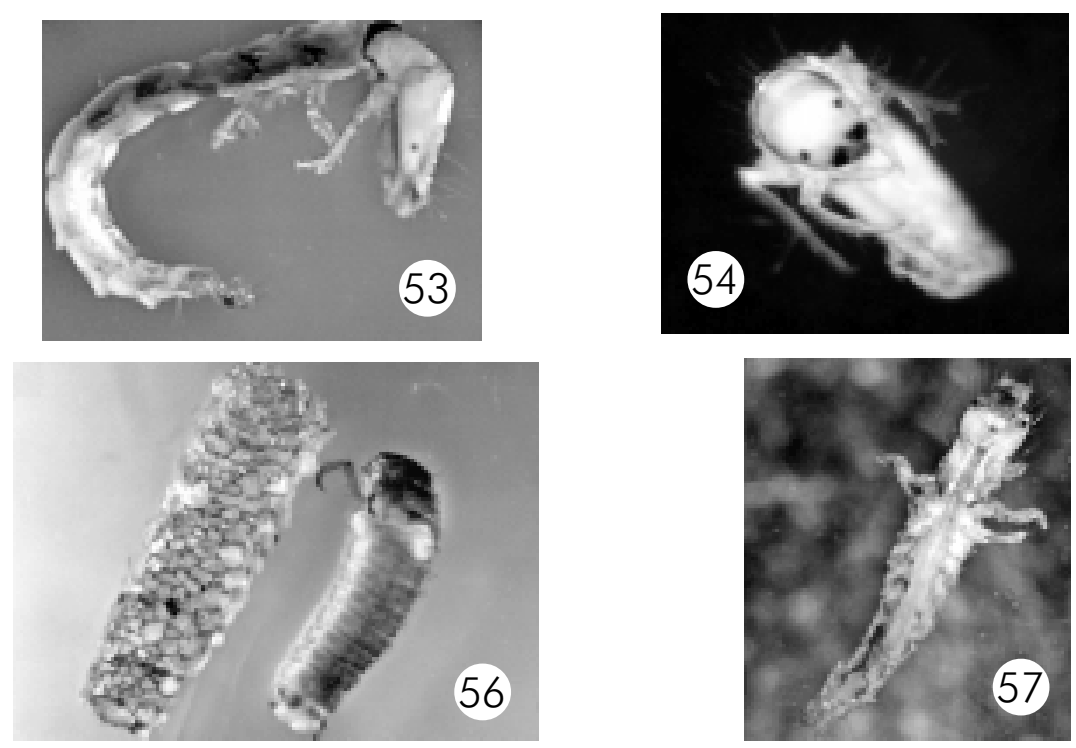

Revista Respuestas - Universidad Francisco de Paula Santander Año 10 No. 1 
Macroinvertebrados del Norte de Santander
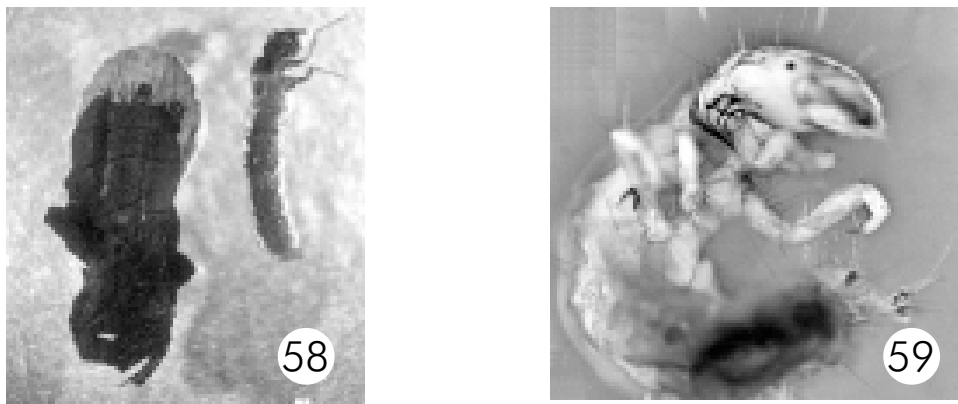

Orden: Tricladida

\begin{tabular}{|c|c|c|c|c|c|c|c|c|c|}
\hline \multirow{2}{*}{\multicolumn{2}{|c|}{ FAMILIA }} & \multicolumn{2}{|c|}{ Cuenca río Zulia } & \multicolumn{2}{|c|}{$\begin{array}{l}\text { Cuenca río } \\
\text { Pamplonita }\end{array}$} & \multicolumn{2}{|c|}{$\begin{array}{l}\text { Cuenca río } \\
\text { Algodonal }\end{array}$} & \multicolumn{2}{|c|}{$\begin{array}{c}\text { Cuenca río } \\
\text { Táchira }\end{array}$} \\
\hline & & $\mathrm{msnm}$ & ${ }^{\circ} \mathrm{C}$ & msnm & ${ }^{\circ} \mathrm{C}$ & msnm & ${ }^{\circ} \mathrm{C}$ & msnm & ${ }^{\circ} \mathrm{C}$ \\
\hline 60 & Planariidae & $\begin{array}{l}1298 \\
1200 \\
279\end{array}$ & $\begin{array}{l}18.7 \\
21.8 \\
23.7\end{array}$ & $\begin{array}{l}2900 \\
2800 \\
2400 \\
2314 \\
2249 \\
2120 \\
1045 \\
719 \\
400 \\
250 \\
160\end{array}$ & $\begin{array}{l}11.5 \\
12.5 \\
14.5 \\
14.5 \\
14.5 \\
17.5 \\
19.5 \\
24 \\
25 \\
33 \\
30\end{array}$ & $\begin{array}{c}1209 \\
970\end{array}$ & $\begin{array}{l}24.8 \\
23.5\end{array}$ & 2435 & 25.1 \\
\hline
\end{tabular}

Orden: Tubificida
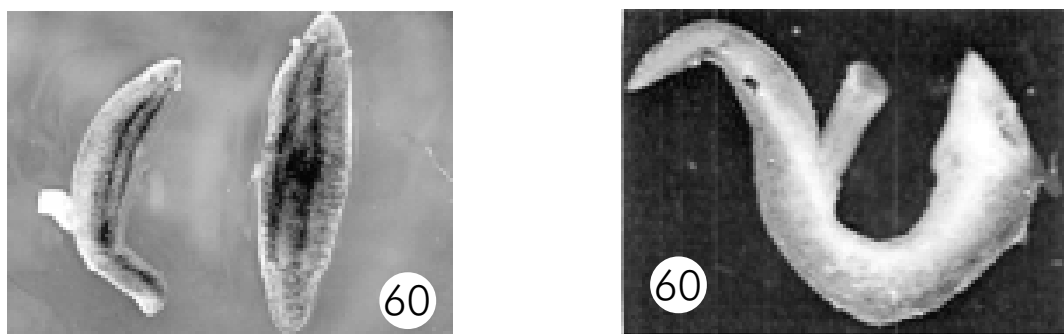

\begin{tabular}{|c|c|c|c|c|c|c|c|c|c|}
\hline \multicolumn{2}{|c|}{ FAMILIA } & \multicolumn{2}{|c|}{ Cuenca río Zulia } & \multicolumn{2}{|c|}{$\begin{array}{l}\text { Cuenca río } \\
\text { Pamplonita }\end{array}$} & \multicolumn{2}{|c|}{$\begin{array}{l}\text { Cuenca río } \\
\text { Algodonal }\end{array}$} & \multicolumn{2}{|c|}{$\begin{array}{l}\text { Cuenca río } \\
\text { Táchira }\end{array}$} \\
\hline & & $\mathrm{msnm}$ & ${ }^{\circ} \mathrm{C}$ & msnm & ${ }^{\circ} \mathrm{C}$ & msnm & ${ }^{\circ} \mathrm{C}$ & msnm & ${ }^{\circ} \mathrm{C}$ \\
\hline 61 & Naididae & $\begin{array}{l}1298 \\
1200 \\
925 \\
904\end{array}$ & $\begin{array}{l}18.7 \\
21.8 \\
23.2 \\
24\end{array}$ & $\begin{array}{l}2900 \\
2800 \\
2400 \\
2314 \\
2249 \\
2120 \\
1045 \\
719 \\
400 \\
250 \\
200 \\
160 \\
100 \\
50\end{array}$ & $\begin{array}{l}11.5 \\
12.5 \\
14.5 \\
14.5 \\
14.5 \\
17.5 \\
19.5 \\
24 \\
25 \\
33 \\
34.5 \\
30 \\
32.5 \\
33.5\end{array}$ & $\begin{array}{l}1379 \\
1392 \\
1310 \\
1233 \\
970\end{array}$ & $\begin{array}{c}22 \\
21 \\
20.2 \\
22 \\
23.5\end{array}$ & $\begin{array}{l}1800 \\
1200\end{array}$ & $\begin{array}{l}27.6 \\
26.5\end{array}$ \\
\hline 62 & Tubificidae & & & $\begin{array}{l}2800 \\
2400 \\
2314 \\
2249 \\
2120 \\
1045 \\
719 \\
400 \\
250 \\
200 \\
160 \\
100 \\
50\end{array}$ & $\begin{array}{l}12.5 \\
14.5 \\
14.5 \\
14.5 \\
17.5 \\
19.5 \\
24 \\
25 \\
33 \\
34.5 \\
30 \\
32.5 \\
33.5\end{array}$ & 1310 & 20.2 & & \\
\hline
\end{tabular}



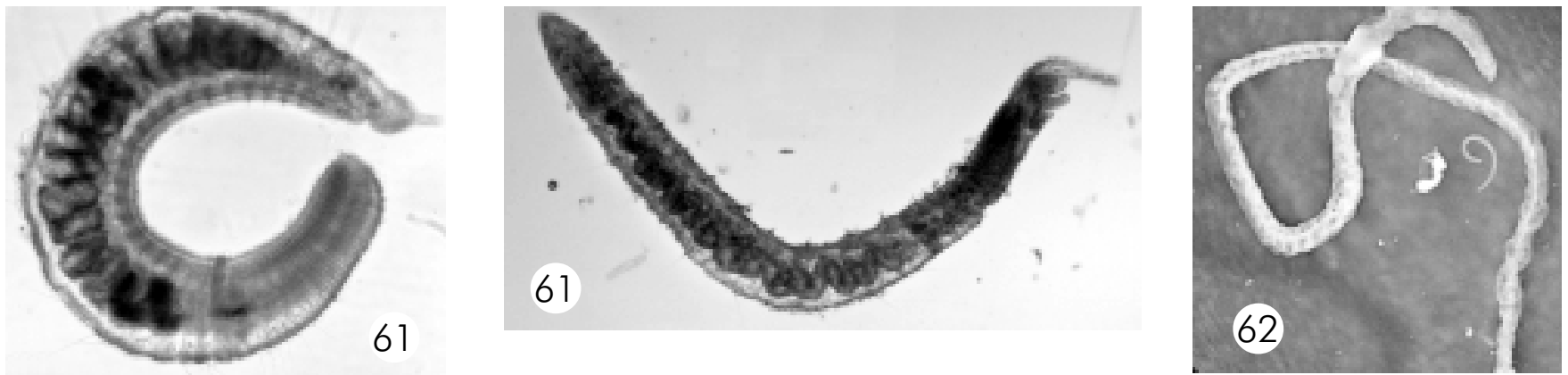

Orden: Unionida

\begin{tabular}{|c|c|c|c|c|c|c|c|c|c|}
\hline \multirow{2}{*}{\multicolumn{2}{|c|}{ FAMILIA }} & \multicolumn{2}{|c|}{$\begin{array}{c}\text { Cuenca río } \\
\text { Zulia }\end{array}$} & \multicolumn{2}{|c|}{$\begin{array}{l}\text { Cuenca río } \\
\text { Pamplonita }\end{array}$} & \multicolumn{2}{|c|}{$\begin{array}{l}\text { Cuenca río } \\
\text { Algodonal }\end{array}$} & \multicolumn{2}{|c|}{$\begin{array}{c}\text { Cuenca río } \\
\text { Táchira }\end{array}$} \\
\hline & & msnm & ${ }^{\circ} \mathrm{C}$ & msnm & ${ }^{\circ} \mathrm{C}$ & msnm & ${ }^{\circ} \mathrm{C}$ & msnm & ${ }^{\circ} \mathrm{C}$ \\
\hline 63 & Sphaeridae & & & $\begin{array}{l}2800 \\
1045\end{array}$ & $\begin{array}{l}12.5 \\
19.5\end{array}$ & & & & \\
\hline
\end{tabular}
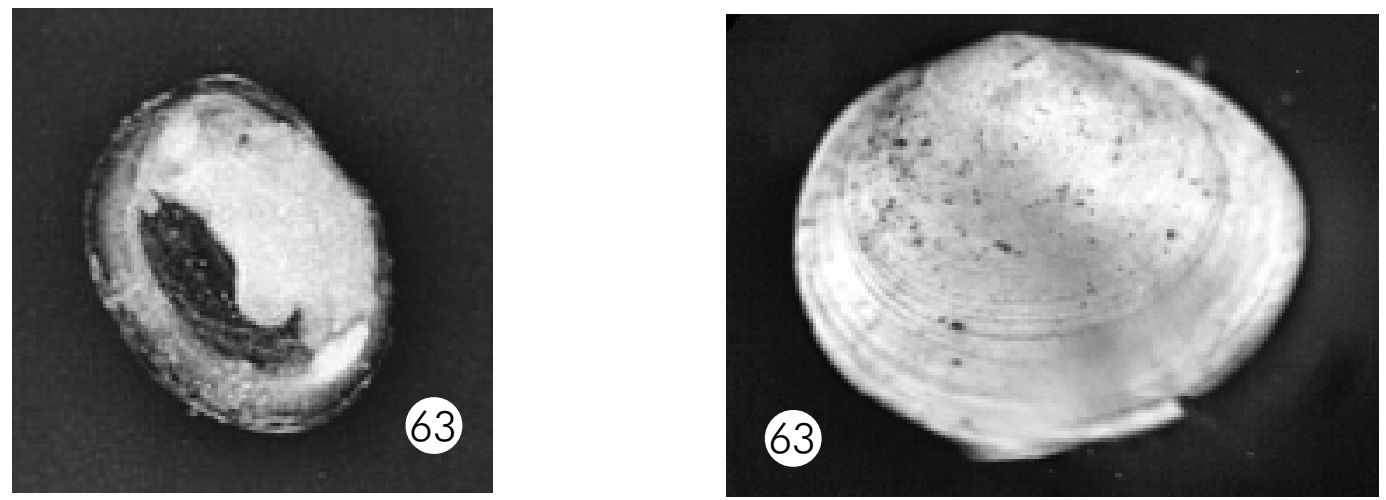

Orden: Gordioidea

\begin{tabular}{|l|l|c|l|c|c|c|c|c|c|}
\hline \multirow{2}{*}{} & \multicolumn{2}{c|}{$\begin{array}{c}\text { Cuenca río } \\
\text { Zulia }\end{array}$} & \multicolumn{2}{c|}{$\begin{array}{c}\text { Cuenca río } \\
\text { Pamplonita }\end{array}$} & \multicolumn{2}{c|}{$\begin{array}{c}\text { Cuenca río } \\
\text { Algodonal }\end{array}$} & \multicolumn{2}{c|}{$\begin{array}{c}\text { Cuenca río } \\
\text { Táchira }\end{array}$} \\
\cline { 3 - 9 } & $\mathrm{msnm}$ & ${ }^{\circ} \mathrm{C}$ & $\mathrm{msnm}$ & ${ }^{\circ} \mathrm{C}$ & $\mathrm{msnm}$ & ${ }^{\circ} \mathrm{C}$ & $\mathrm{msnm}$ & ${ }^{\circ} \mathrm{C}$ \\
\hline 64 & Chordodidae & & & 2800 & 12.5 & & & & \\
& & & 2249 & 14.5 & & & & \\
\end{tabular}

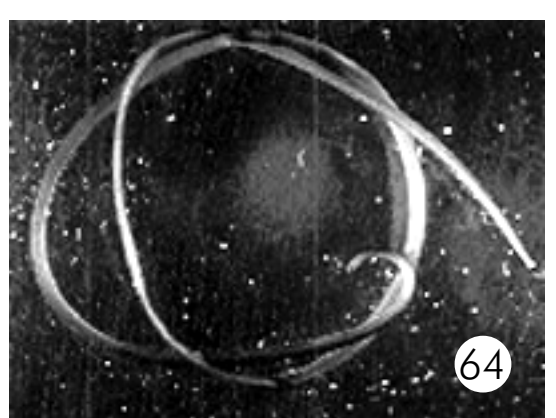


Orden: Rhabditida

\begin{tabular}{|c|c|c|c|c|c|c|c|c|c|}
\hline \multirow{2}{*}{\multicolumn{2}{|c|}{ FAMILIA }} & \multicolumn{2}{|c|}{ Cuenca río Zulia } & \multicolumn{2}{|c|}{$\begin{array}{l}\text { Cuenca río } \\
\text { Pamplonita }\end{array}$} & \multicolumn{2}{|c|}{$\begin{array}{l}\text { Cuenca río } \\
\text { Algodonal }\end{array}$} & \multicolumn{2}{|c|}{$\begin{array}{l}\text { Cuenca río } \\
\text { Táchira }\end{array}$} \\
\hline & & $\mathrm{msnm}$ & ${ }^{\circ} \mathrm{C}$ & msnm & ${ }^{\circ} \mathrm{C}$ & $\mathrm{msnm}$ & ${ }^{\circ} \mathrm{C}$ & $\mathrm{msnm}$ & ${ }^{\circ} \mathrm{C}$ \\
\hline 65 & $\begin{array}{l}\text { Nematodos } \\
\text { Rhabditiformes }\end{array}$ & & & $\begin{array}{l}2800 \\
2400 \\
2314 \\
2249 \\
2120 \\
1045 \\
250 \\
200 \\
160\end{array}$ & $\begin{array}{l}12.5 \\
14.5 \\
14.5 \\
14.5 \\
17.5 \\
19.5 \\
33 \\
34.5 \\
30\end{array}$ & & & & \\
\hline
\end{tabular}
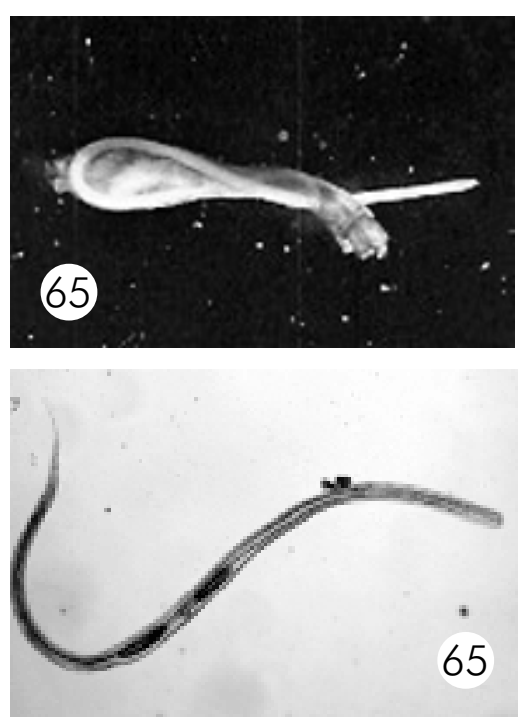

Cada una de las familias presentadas en este artículo se les otorga un puntaje según el BMWP (Biological Monitoring Working Party Score) que debe ser adaptado y modificado acorde a las características del cuerpo de agua, después de que en este se realizan monitoreos consecutivos en un tiempo aproximado de duración de un ciclo de agua. Las puntuaciones de las familias se suman y el número obtenido evalúa la calidad del agua y se puede obtener su representación cartográfica de la siguiente forma:

Cuadro 2. Significado de los valores del índice biológico color a utilizar en representaciones cartográficas.

\begin{tabular}{|c|c|l|c|}
\hline CLASE & VALOR & \multicolumn{1}{|c|}{ SIGNIFICADO } & COLOR \\
\hline I & $>120$ & Aguas limpias & AZUL \\
& $101-120$ & Aguas no contaminadas o no alteradas de modo sensible & \\
\hline II & $61-100$ & Evidentes algunos efectos de contaminación & VERDE \\
\hline III & $36-60$ & Aguas contaminada & AMARILLO \\
\hline IV & $16-35$ & Aguas muy Contaminadas & NARANJA \\
\hline V & $<15$ & Aguas fuertemente contaminadas & ROJO \\
\hline
\end{tabular}




\section{CONCLUSIONES}

Los resultados biológicos permiten establecer el comportamiento histórico de la calidad del agua debido a que las familias de Macroinvertebrados pueden ser sensibles a la contaminación orgánica, lo cual les permite indicar las condiciones que prevalecen y las fluctuaciones que puedan presentarse en un sitio determinado. Si las condiciones son muy fuertes, estas pueden lograr un cambio significativo en la estructura de la comunidad indicadora. La diversidad de familias de Macroinvertebrados en un cuerpo de agua corriente es proporcional a su grado de contaminación, situación que se presenta en la zona estudiada.

Agradecimientos: Lic. Jorge Cuartas

\section{BIBLIOGRAFÍA}

BRINKHURST, R. O y MARCHASE, M.R. Guía para la identificación de Oligoquetos acuáticos continentales de sud y centro América. Argentina. 1991. Colección CLIMAXN.G.2 ${ }^{a}$ ed. 207 p.

CURSO DE INSECTOS INMADUROS. Traducción y adaptación de las claves de Alvah Peterson, Larvae of insects. Edwards Brothers, Inc- Ann Arbor, Michigan. 1960. Universidad del Valle. Angela Martha Rojas.

FERNANDEZ, H.R. y DOMINGUEZ E. Guía para la determinación de los Artropodos Bentónicos Sudamericanos. Universidad Nacional de TucumanArgentina. 2001.282p

MC. CAFFERTY, W..P.. Aquatic Entomology. Science Books International. Boston, Massachussets. 1981. 448 p.

NEEDHAN J., NEEDHAN, Raul. Guía para el estudio de los seres vivos de las aguas dulces. Ed. Reverté. Barcelona. 1982. $131 \mathrm{p}$.

ROLDÁN, Gabriel. Guía para el estudio de los Macroinvertebrados acuáticos del departamento de
Antioquia. Universidad de Antioquia. FEN Colciencias, Edit.Presencia, Bogotá. 1988.

SÁNCHEZ H. Marjorie J., et al. Macroinvertebrados acuáticos como bioindicadores de la calidad del agua de la zona media del río Pamplonita. Universidad Francisco de Paula Santander. 2001. 204p.

\section{Estudio}

Limnológico de la Zona Alta del río Pamplonita. Universidad Francisco de Paula Santander. 2004. $127 p$.

Recibido: Abril 6 de 2005

Aceptado: Mayo 27 de 2005 